\title{
Antenna Array for Passive Radar: Configuration Design and Adaptive Approaches to Disturbance Cancellation
}

\author{
Michelangelo Villano, ${ }^{1}$ Fabiola Colone, ${ }^{2}$ and Pierfrancesco Lombardo ${ }^{2}$ \\ ${ }^{1}$ German Aerospace Center (DLR), Microwaves and Radar Institute, Oberpfaffenhofen, 82234 Wessling, Germany \\ ${ }^{2}$ Department of Information Engineering, Electronics and Telecommunications (DIET), University of Rome "La Sapienza", \\ Via Eudossiana 18, 00184 Rome, Italy
}

Correspondence should be addressed to Fabiola Colone; fabiola.colone@diet.uniromal.it

Received 5 April 2013; Accepted 4 September 2013

Academic Editor: Ulrich Nickel

Copyright (C) 2013 Michelangelo Villano et al. This is an open access article distributed under the Creative Commons Attribution License, which permits unrestricted use, distribution, and reproduction in any medium, provided the original work is properly cited.

We consider the selection of an antenna array configuration, composed of a small number of omnidirectional elements, to be exploited for passive radar sensors. Based on properly identified pattern characteristics and design criteria for practical applications, a suitable planar configuration is selected that allows both angular selectivity and direct signal attenuation. The selected configuration is further optimized in terms of sidelobe level by resorting to appropriate amplitude tapering. Moreover, three different approaches are investigated for antenna-based adaptive disturbance cancellation, and a comparative performance analysis is carried out. Simulation results show that an effective clutter suppression is obtained if the direct signal from the transmitter is attenuated by means of spatial adaptive cancellation, and the multipath echoes from stationary obstacles are removed by means of temporal adaptive cancellation. In particular, the approach based on the Sidelobe Canceller is shown to yield good performance while requiring a limited system complexity.

\section{Introduction}

Passive radar systems are specific variants of bistatic radar that exploit existing transmitters as "illuminators of opportunity". The main advantages of bistatic radar are that the receiver is far less vulnerable to electronic counter measures (ECM) and that bistatic operation has counter stealth properties. Moreover, passive radar systems have much lower cost, as they do not need a dedicated transmitter [1-3].

In spite of all these advantages, which make passive radar attractive for a broad range of applications, they must cope with the use of nonoptimized waveforms and with a strong direct signal from the transmitter of opportunity that typically operates in continuous wave (CW) mode. This strong disturbance must be appropriately cancelled, together with its reflections from stationary obstacles in the scene (clutter/multipath) to improve detection performance. Directive antennas, characterized by a high Front-to-Back Ratio (FBR), are usually employed to attenuate the direct signal. Its residuals are then typically removed by means of temporal adaptive clutter cancellation together with the clutter contribution [4-6]. However, the use of a directive antenna provides only a limited angular coverage for the radar surveillance and does not allow to cover a very wide air space region.

This drawback can be avoided by using an antenna array composed of a set of omnidirectional elements in the plane, in place of a dedicated directive surveillance antenna [79]. As long as the elements are properly spaced, a directive pattern may be synthesized by jointly processing the signals received at each element. This also allows the beam to be electronically steered in all directions or, better, a set of directional beams to be generated that globally cover the whole air space of interest. Obviously this would require the availability of multiple coherent receiving channels. However, in this case the same array might be used to collect the transmitted signal by synthesizing a beam pointed toward the transmitter of opportunity. This represents a viable solution if several dislocated illuminators of opportunity should be exploited. 
To keep the system cost and its complexity low, proper array configurations should be designed and able to provide good performance using a limited number of elements [10]. In this paper, we describe effective criteria to identify a suitable antenna array configuration for passive radar. According to the proposed strategy, the array is designed so that it yields reasonable characteristics for the resulting antenna pattern ( $3 \mathrm{~dB}$ aperture, sidelobe level) while satisfying a few constraints that allow the $360^{\circ}$ operation.

Furthermore, the considered planar array may be provided with spatial adaptivity, so that the clutter/multipath cancellation filter can benefit from space-time adaptivity.

The benefits of antenna-based spatial adaptivity have been shown in the literature with reference to different passive radar applications. A clear demonstration of potentiality of the spatial adaptivity is reported in [11] for a GSM-based passive radar for medium range surveillance, in [12] for an FM radio-based passive radar, and in [13] for a specific passive radar application in the HF frequency bandwidth. In [14], the space-time adaptive beamformer based on constrained least mean squares algorithm has been exploited. Even for the reference signal recovery, adaptive techniques can be used to protect the system against multipath contributions. For example, in [15] the joint exploitation of spatial and temporal degrees of freedom is considered to obtain a multipath-free version of the reference signal.

Therefore, in this paper, we investigate different techniques for antenna-based adaptive disturbance cancellation to be applied before the standard temporal cancellation filter. The comparative analysis allows to identify the main benefits of the different approaches and to select the most suitable space-time processing scheme. Specifically, the simulation results, obtained for an FM radio-based passive radar case, show that the approach based on the Sidelobe Canceller followed by the temporal extensive cancellation algorithm (ECA) [4] yields good performance while requiring a limited system complexity.

The paper is organized as follows. In Section 2, the array design strategy is described, and a suitable antenna array configuration is selected by trading off the achievable performance for the expected system complexity and cost. The selected array configuration is further optimized in Section 3 where a proper amplitude tapering strategy is adopted to control the sidelobe level of the resulting pattern. Section 4 briefly summarizes the considered techniques for antenna-based adaptive disturbance cancellation; moreover, an effective approach is introduced for the synthesis of the reference antenna beam that is exploited to collect the signal from the transmitter. The comparative performance analysis is presented in Section 5 where the results are reported for an FM radio-based passive radar scenario. Finally, our conclusions are drawn in Section 6.

\section{Antenna Array Configuration Analysis}

The first step of our work consists in identifying an appropriate configuration for the array of antennas that provides good performance using a limited number of elements (to keep the low cost characteristic). Specifically, we refer to 2-dimensional (planar) array configurations. A reasonable criterion to select a planar array configuration may involve the evaluation of some significant pattern parameters, such as the $3 \mathrm{~dB}$ aperture and the peak-to-side lobe ratio (PSLR). It is also reasonable to restrict the choice to those configurations, for which the expected (angle estimation) performance does not depend on the Direction of Arrival (DoA) of the signal. In particular, it is possible to refer to the estimation accuracy achievable by the Generalized Likelihood (GL) estimation.

2.1. GL DoA Estimation and Its Accuracy. Let us consider a planar array of $N$ elements and let $\left(x_{i}, y_{i}\right)$ and $g_{i}(\theta)$ be the coordinates and the azimuth pattern of the $i$ th element, respectively, being $\theta$ the angle formed with the positive $y$ axis. Then, the target steering vector $\mathbf{s}_{\boldsymbol{\theta}}(\theta)$ can be written as

$$
\mathbf{s}_{\boldsymbol{\theta}}(\theta)=\left[\begin{array}{c}
g_{1}(\theta) \exp \left\{j \frac{2 \pi}{\lambda}\left(x_{1} \sin \theta+y_{1} \cos \theta\right)\right\} \\
\vdots \\
g_{i}(\theta) \exp \left\{j \frac{2 \pi}{\lambda}\left(x_{i} \sin \theta+y_{i} \cos \theta\right)\right\} \\
\vdots \\
g_{N}(\theta) \exp \left\{j \frac{2 \pi}{\lambda}\left(x_{N} \sin \theta+y_{N} \cos \theta\right)\right\}
\end{array}\right]
$$

where $\lambda$ is the wavelength of the received signal.

The signals received at a given time by the elements of the array may be collected in an $N$-element column vector $\mathbf{x}$, called snapshot, and given by

$$
\mathbf{x}=a \mathbf{s}_{\boldsymbol{\theta}}\left(\theta_{T}\right)+\mathbf{v}, \quad \mathbf{v}=\left[\begin{array}{c}
v_{1} \\
\vdots \\
v_{i} \\
\vdots \\
v_{N}
\end{array}\right]
$$

where $\theta_{T}$ is the signal DoA, $a$ is the complex amplitude of the received signal, which does not include the antenna gain, and $\mathbf{v}$ is the disturbance.

We assume for $\mathbf{x}$ a Gaussian distribution, whose expected value and covariance matrix are given by

$$
\begin{gathered}
\overline{\mathbf{x}}=E\{\mathbf{x}\}=\operatorname{\gamma as}_{\boldsymbol{\theta}}\left(\theta_{T}\right), \\
\gamma= \begin{cases}0, & \mathrm{Hp} \cdot \mathrm{H}_{0}(\text { absence of target }) \\
1, & \mathrm{Hp} \cdot \mathrm{H}_{1}(\text { presence of target }),\end{cases} \\
\mathbf{M}=E\left\{(\mathbf{x}-\overline{\mathbf{x}})(\mathbf{x}-\overline{\mathbf{x}})^{H}\right\}=\sigma_{n}^{2} \mathbf{I},
\end{gathered}
$$

respectively, where $\sigma_{n}^{2}$ is the noise variance and $\mathbf{v}^{H}$ denotes the Hermitian transpose of vector $\mathbf{v}$. The DoA $\theta_{T}$ of a monochromatic signal, whose complex amplitude $a$ is unknown, may be estimated by maximizing the Generalized Likelihood, as in

$$
\widehat{\theta}_{T}=\arg \min _{\theta}\left\|\mathbf{x}-\frac{\mathbf{s}_{\boldsymbol{\theta}}^{H}(\theta) \mathbf{x s}_{\boldsymbol{\theta}}(\theta)}{\mathbf{s}_{\boldsymbol{\theta}}^{H}(\theta) \mathbf{s}_{\boldsymbol{\theta}}(\theta)}\right\|^{2},
$$


where the operators $\|\mathbf{v}\|$ denote the Euclidean norm of vector $\mathbf{v}$, while the notation arg $\min _{\theta} f(\theta)$ denotes the value of $\theta$ for which $f(\theta)$ is minimum.

In particular, it can be shown that the resulting estimation accuracy is given by (see, e.g., [16])

$$
\begin{aligned}
\sigma_{\theta_{T}}\left(\theta_{T}\right) & =\sqrt{E\left\{\left|\hat{\theta}_{T}-\theta_{T}\right|^{2}\right\}-\left|E\left\{\hat{\theta}_{T}-\theta_{T}\right\}\right|^{2}} \\
& =\left\{2 \frac{|a|^{2}}{\sigma_{n}^{2}}\left[\left\|\dot{\mathbf{s}}_{\boldsymbol{\theta}}\left(\theta_{T}\right)\right\|^{2}-\frac{\left|\dot{\mathbf{s}}_{\boldsymbol{\theta}}^{H}\left(\theta_{T}\right) \mathbf{s}_{\boldsymbol{\theta}}\left(\theta_{T}\right)\right|^{2}}{\left\|\mathbf{s}_{\boldsymbol{\theta}}\left(\theta_{T}\right)\right\|^{2}}\right]\right\}^{-1 / 2},
\end{aligned}
$$

where $\dot{\mathbf{s}}_{\boldsymbol{\theta}}\left(\theta_{T}\right)$ is the first-order derivative of $\mathbf{s}_{\boldsymbol{\theta}}\left(\theta_{T}\right)$ with respect to $\theta_{T}$.

2.2. Omnidirectional Antennas and Symmetry Conditions. In case all the antennas are omnidirectional, for example,

$$
g_{i}(\theta)=g, \quad i=1 \cdots N
$$

the target steering vector $\mathbf{s}_{\boldsymbol{\theta}}(\theta)$ and its first-order derivative with respect to $\theta$ may be rewritten as

$$
\dot{\mathbf{s}}_{\boldsymbol{\theta}}(\theta)=\left[\begin{array}{c}
g \exp \left\{j \frac{2 \pi}{\lambda}\left(x_{1} \sin \theta+y_{1} \cos \theta\right)\right\} \\
g \exp \left\{j \frac{2 \pi}{\lambda}\left(x_{i} \sin \theta+y_{i} \cos \theta\right)\right\} \\
\vdots \\
g \exp \left\{j \frac{2 \pi}{\lambda}\left(x_{N} \sin \theta+y_{N} \cos \theta\right)\right\}
\end{array}\right],
$$

respectively, and the quantities $\left\|\dot{\mathbf{s}}_{\boldsymbol{\theta}}\left(\theta_{T}\right)\right\|^{2},\left|\dot{\mathbf{s}}_{\boldsymbol{\theta}}^{H}\left(\theta_{T}\right) \mathbf{s}_{\boldsymbol{\theta}}\left(\theta_{T}\right)\right|^{2}$, and $\left\|\mathbf{s}_{\boldsymbol{\theta}}\left(\theta_{T}\right)\right\|^{2}$ in (5) may be rewritten as follows:

$$
\begin{gathered}
\left\|\dot{\mathbf{s}}_{\boldsymbol{\theta}}\left(\theta_{T}\right)\right\|^{2}=g^{2} \frac{4 \pi^{2}}{\lambda^{2}} \sum_{i=1}^{N}\left(x_{i} \cos \theta_{T}-y_{i} \sin \theta_{T}\right)^{2}, \\
\left|\dot{\mathbf{s}}_{\boldsymbol{\theta}}^{H}\left(\theta_{T}\right) \mathbf{s}_{\boldsymbol{\theta}}\left(\theta_{T}\right)\right|^{2}=g^{4} \frac{4 \pi^{2}}{\lambda^{2}}\left[\sum_{i=1}^{N}\left(x_{i} \cos \theta_{T}-y_{i} \sin \theta_{T}\right)\right]^{2}, \\
\left\|\mathbf{s}_{\boldsymbol{\theta}}\left(\theta_{T}\right)\right\|^{2}=g^{2} N .
\end{gathered}
$$

Therefore, the estimation accuracy in the case of omnidirectional antennas is given by

$$
\sigma_{\theta_{T}}\left(\theta_{T}\right)=\left[2 \frac{|a|^{2}}{\sigma_{n}^{2}} g^{2} \frac{4 \pi^{2}}{\lambda^{2}} f\left(\theta_{T}\right)\right]^{-1 / 2},
$$

where

$$
\begin{aligned}
f\left(\theta_{T}\right)= & \sum_{i=1}^{N}\left(x_{i} \cos \theta_{T}-y_{i} \sin \theta_{T}\right)^{2} \\
& -\frac{1}{N}\left[\sum_{i=1}^{N}\left(x_{i} \cos \theta_{T}-y_{i} \sin \theta_{T}\right)\right]^{2} .
\end{aligned}
$$

If the following sets of symmetry conditions, namely,

$$
\begin{gathered}
\sum_{i=1}^{N} x_{i}=0, \quad \sum_{i=1}^{N} y_{i}=0, \\
\sum_{i=1}^{N} x_{i}^{2}=\sum_{i=1}^{N} y_{i}^{2}, \quad \sum_{i=1}^{N} x_{i} y_{i}=0
\end{gathered}
$$

hold, the expression of the estimation accuracy in (9) may be further simplified. In particular, the estimation accuracy is no longer dependent on the signal DoA.

Holding (11), in fact, (10) becomes

$$
f\left(\theta_{T}\right)=\sum_{i=1}^{N}\left(x_{i} \cos \theta_{T}-y_{i} \sin \theta_{T}\right)^{2} .
$$


Then, $f\left(\theta_{T}\right)$ can be expanded and, holding (12), simplified as follows:

$$
\begin{aligned}
f\left(\theta_{T}\right)= & \sum_{i=1}^{N}\left(x_{i} \cos \theta_{T}-y_{i} \sin \theta_{T}\right)^{2} \\
= & \cos ^{2} \theta_{T} \sum_{i=1}^{N} x_{i}^{2}+\sin ^{2} \theta_{T} \sum_{i=1}^{N} y_{i}^{2} \\
& -2 \cos \theta_{T} \sin \theta_{T} \sum_{i=1}^{N} x_{i} y_{i} \\
= & \left(\cos ^{2} \theta_{T}+\sin ^{2} \theta_{T}\right) \sum_{i=1}^{N} x_{i}^{2} \\
= & \sum_{i=1}^{N} x_{i}^{2}=\sum_{i=1}^{N} y_{i}^{2}=\frac{1}{2} \sum_{i=1}^{N}\left(x_{i}^{2}+y_{i}^{2}\right)=\frac{1}{2} \sum_{i=1}^{N} R_{i}^{2},
\end{aligned}
$$

where $R_{i}$ is the Euclidean distance of the $i$ th element from the origin of the reference system.

2.3. Symmetric Configurations. Those configurations for which the symmetry conditions (11)-(12) hold, are from now on referred to as symmetric configurations. We are indeed interested in these configurations because the considered application does not require specific directions to be preferred.

The next step of our configuration analysis, therefore, consists in identifying some symmetric configurations, characterized by a relatively small number of elements. We first observe that the two-element arrays cannot jointly satisfy (11) and (12). On the other hand, the number $N$ of elements is upper-limited to 10 in order to make the system both compact and low-cost.

Let us denote by $R$ the maximum distance of an element of the array from the origin of the reference system or, equivalently, the radius of the smallest circle containing all the array elements.

It is possible to list different classes of symmetric configurations (in brackets the number of elements required to form such a configuration), namely,

(i) circular configurations without central element $(N \geq$ 3 ), where $N$ elements are uniformly arranged on the circumference of a circle of radius $R$, centered at the origin of the reference system;

(ii) circular configurations with central element $(N \geq 4)$, where $(N-1)$ elements are uniformly arranged on the circumference of a circle of radius $R$, and one element is located in the center of such a circle;

(iii) triangular configurations without central element $(N=$ $3 k$ ), where $N$ elements are uniformly arranged on the edges of an equilateral triangle, inscribed in a circle of radius $R$. The trivial case $k=1$ is not considered, as it belongs to the class of circular configurations without central element;

(iv) triangular configurations with central element $(N=$ $3 k+1)$, where $(N-1)$ elements are uniformly arranged on the edges of an equilateral triangle, inscribed in a circle of radius $R$, and one element is located in the center of such a circle. The trivial case $k=1$ is not considered, as it belongs to the class of circular configurations with central element;

(v) squared configurations without central element $(N=$ $4 k$ ), where $N$ elements are uniformly arranged on the edges of a square, inscribed in a circle of radius $R$.The trivial case $k=1$ is not considered, as it belongs to the class of circular configurations without central element;

(vi) squared configurations with central element $(N=4 k+$ $1)$, where $(N-1)$ elements are uniformly arranged on the edges of a square, inscribed in a circle of radius $R$, and one element is located in the center of such a circle. The trivial case $k=1$ is not considered, as it belongs to the class of circular configurations with central element;

(vii) $Y$ configurations $(N=3 k, N=3 k+1)$, where the elements are uniformly arranged on the radii joining the vertices of an equilateral triangle, inscribed in a circle of radius $R$, to the center of the circle itself. The trivial case $k=1$ is not considered, as it belongs to the first two classes of configurations;

(viii) X configurations $(N=4 k, N=4 k+1)$, where the elements are uniformly arranged on the radii joining the vertices of a square, inscribed in a circle of radius $R$, to the center of the circle itself. The trivial case $k=1$ is not considered, as it belongs to the first two classes of configurations.

In Table 1, the symmetric configurations belonging to the above mentioned classes are represented for different numbers $N$ of antenna elements. Moreover, for each class, a marker plot is defined that will be used in the following figures (see last row of Table 1).

While our study is focused on planar array configurations, we should mention that alternative solutions could be considered based on combinations of three (or more) linear arrays covering adjacent $120^{\circ}$ (or less) sectors to provide the $360^{\circ}$ operation. However, such choice would yield a number of drawbacks that should be taken into account for the considered application. In particular,

(i) the resulting system would be a combination of three (or more) separate systems that need to be synchronized and disciplined to manage possible common detections performed in adjacent/overlapped angular sectors;

(ii) the resulting performance would be a function of the target DoA because each linear array has characteristics that change with the pointing angle (e.g., the $3 \mathrm{~dB}$ aperture). In this regard, we recall that the symmetric array configurations selected in our study guarantee the estimation accuracy to be independent of the target DoA so that the same performance could be in principle obtained over the $360^{\circ}$ coverage; 
TABLE 1: Symmetric configurations belonging to the different classes for $N<10$.

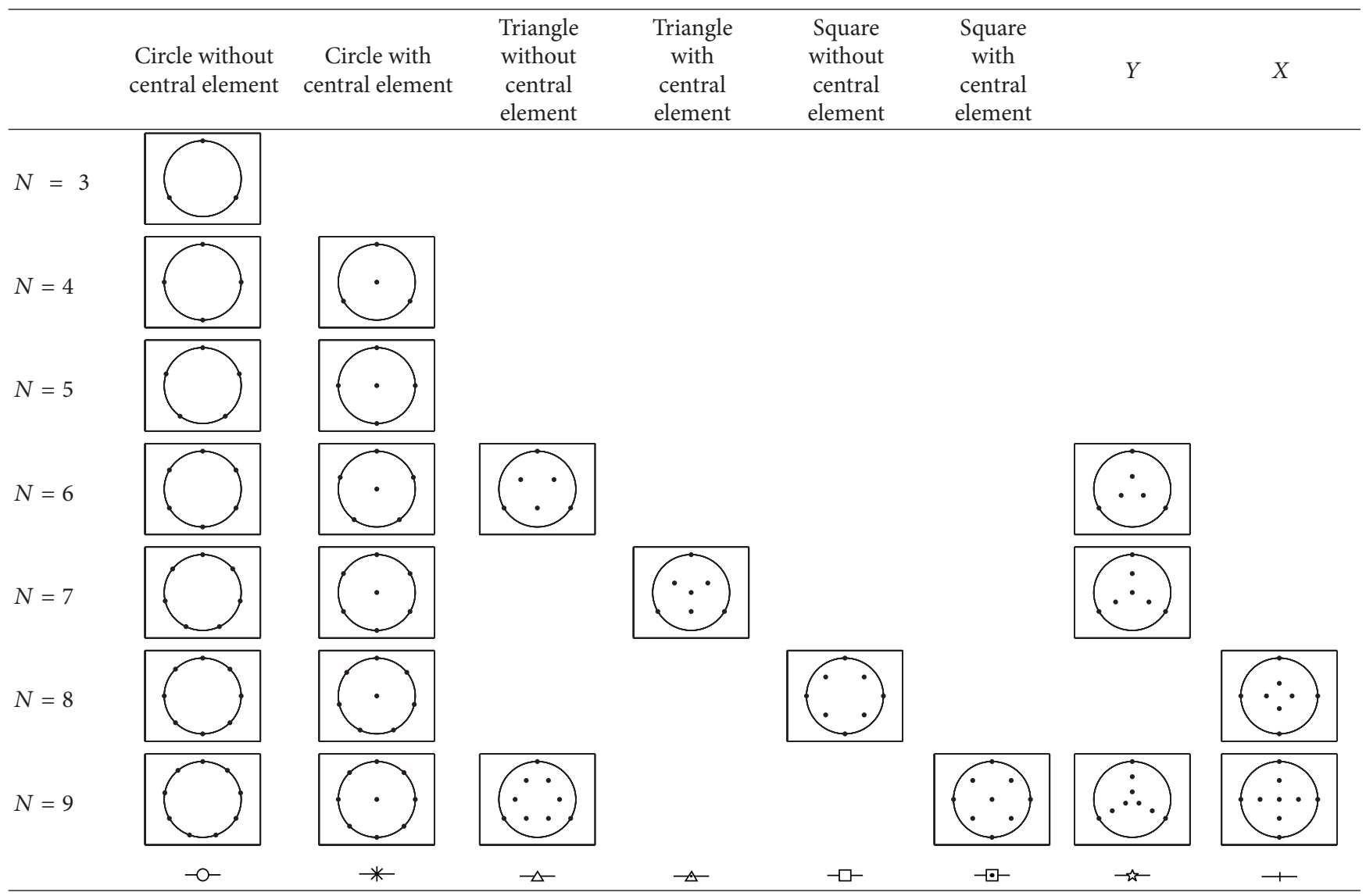

(iii) the problem discussed at point (i) is exacerbated when each array suffers of severe angular ambiguities; to reduce this effect directive antenna elements should be employed to build up each array (e.g., Yagi or Log Periodic antennas could be adopted in the FM radio band). However, this would stress the effect described in (ii). Moreover, using this approach, the overall system would be bulkier and characterized by a much higher cost.

For the above reasons, the symmetric planar array configurations sketched in Table 1 have been preferred and their characteristics are compared in the following subsection with the aim to identify the most suitable solution for the considered application.

\subsection{Pattern Characteristics and Array Configuration Selection.} The selection of the most appropriate configuration for passive radar applications involves the choice of the number $N$ of antenna elements, the identification of its shape, and the determination of the array radius $R$.

So far we have established the conditions for which the DoA estimation accuracy is not dependent on the DoA itself and identified some configurations that satisfy these conditions. In the present subsection, we deal with the selection of a suitable configuration among different symmetric ones.
First of all, it is of interest to highlight the relationship between the GL DoA estimation, as defined in (4), and the array pattern of the configuration. Expanding the expression of the estimated DoA in (4) as

$$
\begin{aligned}
& \widehat{\theta}_{T}=\arg \min _{\theta}\left\|\mathbf{x}-\frac{\mathbf{s}_{\boldsymbol{\theta}}^{H}(\theta) \mathbf{x s}_{\boldsymbol{\theta}}(\theta)}{\mathbf{s}_{\boldsymbol{\theta}}^{H}(\theta) \mathbf{s}_{\boldsymbol{\theta}}(\theta)}\right\|^{2} \\
& =\arg \min _{\theta}\left\{\|\mathbf{x}\|^{2}+\frac{\left\|\mathbf{s}_{\boldsymbol{\theta}}^{H}(\theta) \mathbf{x s}_{\boldsymbol{\theta}}(\theta)\right\|^{2}}{\left\|\mathbf{s}_{\boldsymbol{\theta}}(\theta)\right\|^{4}}-\mathbf{x}^{H} \frac{\mathbf{s}_{\boldsymbol{\theta}}^{H}(\theta) \mathbf{x s}_{\boldsymbol{\theta}}(\theta)}{\left\|\mathbf{s}_{\boldsymbol{\theta}}(\theta)\right\|^{2}}\right. \\
& \left.-\left(\frac{\mathbf{s}_{\boldsymbol{\theta}}^{H}(\theta) \mathbf{x s}_{\theta}(\theta)}{\left\|\mathbf{s}_{\boldsymbol{\theta}}(\theta)\right\|^{2}}\right)^{H} \mathbf{x}\right\} \\
& =\arg \min _{\theta}\left\{\|\mathbf{x}\|^{2}+\frac{\left|\mathbf{s}_{\boldsymbol{\theta}}^{H}(\theta) \mathbf{x}\right|^{2}}{\left\|\mathbf{s}_{\boldsymbol{\theta}}(\theta)\right\|^{2}}-\frac{\left|\mathbf{s}_{\boldsymbol{\theta}}^{H}(\theta) \mathbf{x}\right|^{2}}{\left\|\mathbf{s}_{\boldsymbol{\theta}}(\theta)\right\|^{2}}-\frac{\left|\mathbf{s}_{\boldsymbol{\theta}}^{H}(\theta) \mathbf{x}\right|^{2}}{\left\|\mathbf{s}_{\boldsymbol{\theta}}(\theta)\right\|^{2}}\right\} \\
& =\arg \max _{\theta}\left|\mathbf{s}_{\boldsymbol{\theta}}^{H}(\theta) \mathbf{x}\right|^{2},
\end{aligned}
$$

it is possible to better understand what GL estimation means in the specific case of symmetric configurations; for each direction $\theta$, the signals received by the $N$ antennas are properly phase-shifted and summed; then the estimated DoA 
is that for which the square modulus of such a sum is maximum. In other words, in this specific case, the GL DoA estimation consists in synthesizing infinite beams, pointed to all the directions, being the output of the estimate of the direction, for which the intensity of the signal received by the corresponding synthesized beam is maximum.

The GL estimation accuracy is therefore directly related to the array pattern characteristics, and a suitable configuration may be selected among several ones by evaluating the pattern characteristics themselves. It would not be appropriate to select the configuration for which the estimation accuracy, as defined in (5) or (9), is maximum, because such expression is significant only in the absence of ambiguities, namely, when grating lobes do not occur. A parameter related to the presence/absence of grating lobes, such as the PSLR, has therefore to be considered together with the estimation accuracy. Moreover, since this latter parameter is directly related to the pattern main lobe width, the $3 \mathrm{~dB}$ aperture may be considered in place of the accuracy, which leads us to consider two parameters characteristics of the antenna array pattern.

The $3 \mathrm{~dB}$ aperture and the PSLR have been plotted in Figure 1 as a function of the ratio $R / \lambda$ of the array radius $R$ to the wavelength of the received signal $\lambda$, so that a visual comparison can be performed. Specifically, Figures 1(a)-1(g) are obtained by progressively increasing the number of array elements from $N=3$ to $N=9$ and reporting, for each case, only the applicable configuration classes. The adopted markers are described in Table 1 . As both the considered pattern parameters vary with the pointing direction, the worst case is reported, that is, the maximum $3 \mathrm{~dB}$ aperture and the minimum PSLR.

A careful observation of the plots in Figure 1 shows that it is not possible to synthesize array patterns characterized by both a narrow beamwidth and a satisfactory PSLR by using less than $N=8$ elements. At the same time, the introduction of a ninth element does not lead to a significant improvement of the pattern characteristics. We also notice that the choice of $N=8$ antenna elements allows the exploitation of commercial low-cost components to build up the multichannel radar receiver. Among the 8-element configuration, the circular configuration with central element, represented in Figure 2, is particularly attractive when the radius $R$ of the planar array is selected to be equal to $0.44 \lambda$, as the resulting pattern is characterized by both a narrow beamwidth and a reasonable PSLR, namely, $13 \mathrm{~dB}$.

When the sources of radar transmission are FM radio stations, being the wavelength of the signal comprised between $\lambda_{\text {min }}=2.78 \mathrm{~m}(108 \mathrm{MHz})$ and $\lambda_{\text {max }}=3.41 \mathrm{~m}(88 \mathrm{MHz})$, the radius of the array has to be chosen as a compromise value, with special attention to $\lambda_{\text {min }}$.

As a final remark, we notice that the previous analysis of the pattern characteristics has been conducted by assuming that there is no coupling between the array elements. In practice, such coupling effect exists and might significantly modify the resulting antenna pattern. Moreover, it is expected to become more severe as the array radius, and consequently the elements spacing, decreases. Nevertheless, the criteria adopted to identify a suitable antenna array configuration still hold; therefore, the proposed design strategy can still be applied against a more realistic model of the received signal to include the coupling effect as well as its possible compensation according to proper models. Finally, we should mention that, despite the symmetry of the considered array configurations, the coupling effect between different pairs of neighboring elements can be different [7]; however, this is due to nonidealities of array elements, minor errors in array geometry, and nearby obstacles that cannot be taken into account at the design stage but should be measured and compensated for after its assembling.

\section{Side Lobe Control by Central Element Tapering}

It is well known that it is generally possible to synthesize an array pattern with a higher PSLR by applying appropriate tapering to the single array elements. In the general case, the application of a taper function intrinsically destroys the uniform angular performance. Special considerations apply with reference to the selected configuration, which is made up by eight elements, seven of which are uniformly distributed over a circumference and one of which is located in the center of the same circumference. In fact, in this case, there is a simple way to apply something similar to a taper function by weighting differently the array central element and the circle of seven elements. Specifically, it is of interest to determine whether a higher PSLR may be obtained, by properly tapering only the central element.

By letting the taper of the central element $\alpha$ vary over a large enough interval, being unitary the tapers of all the other elements, the PSLR has been evaluated and plotted in Figure 3 as a function of $\alpha$. The value of taper which maximizes the PSLR is $\alpha=1.98$. The PSLR of the synthesized pattern is now greater than $20 \mathrm{~dB}$ (see Figure 4), while there is only a slight broadening of the antenna pattern main lobe (the $3 \mathrm{~dB}$ beamwidth is about $4^{\circ}$ broader than that obtained without tapering). This tapered configuration is especially effective when used for passive radar, since it provides up to $7 \mathrm{~dB}$ improvement in the direct signal rejection, when the main beam is not steered $180^{\circ}$ away from the transmitter of opportunity.

\section{Adaptive Disturbance Cancellation Techniques}

As the received power of the direct signal is usually many tens of $\mathrm{dBs}$ greater than the power received from a target echo, even using amplitude tapering, the direct signal attenuation might not be sufficient, and an adaptive temporal cancellation is typically used to remove the residual direct signal and the clutter, in order to provide the desired detection performance. The adaptive temporal cancellation is based on the principle that in the passive coherent location (PCL) environment, the disturbance includes the direct signal from the bistatic transmitter and multipath replicas of the direct signal, generated by its reflection from stationary obstacles, which are generally referred to as clutters. In contrast, the useful signal 

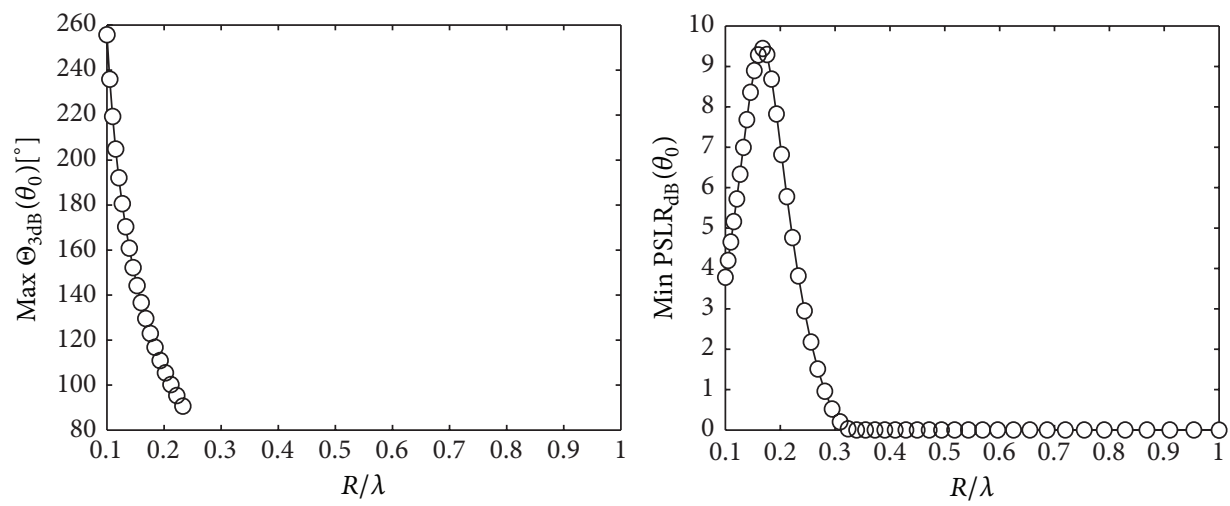

(a)
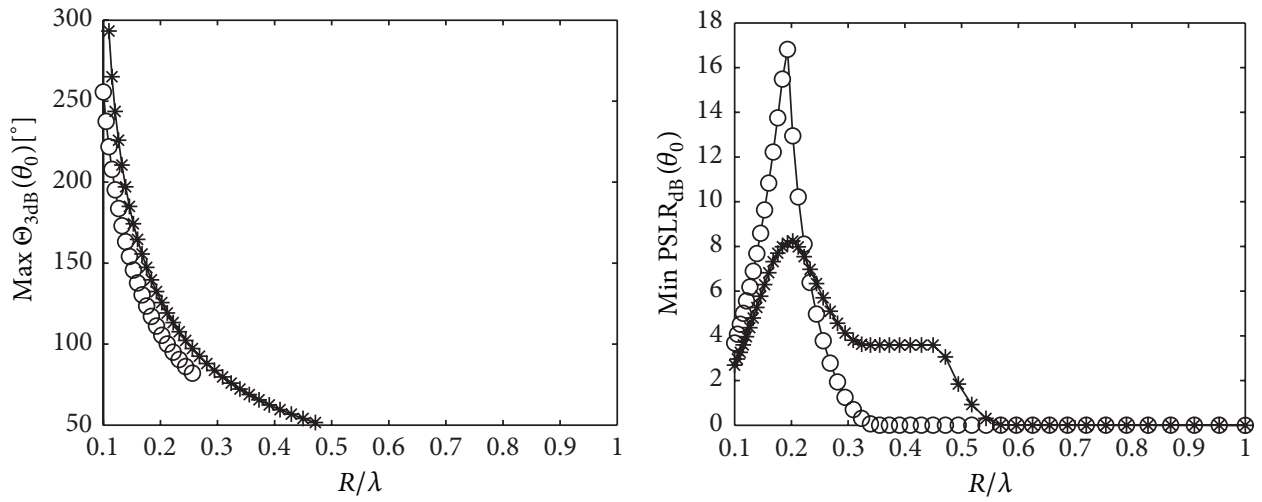

(b)
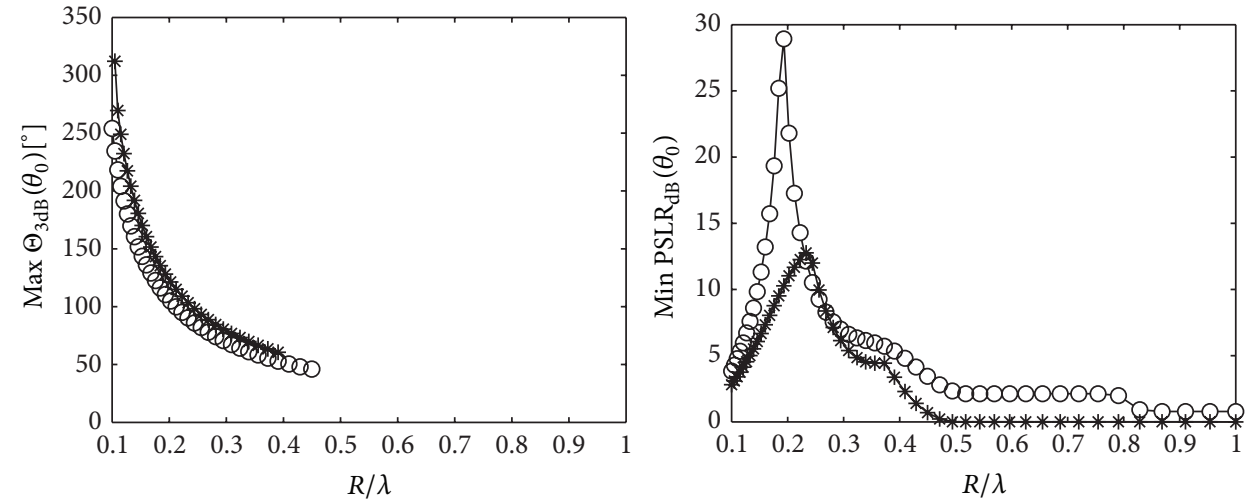

(c)
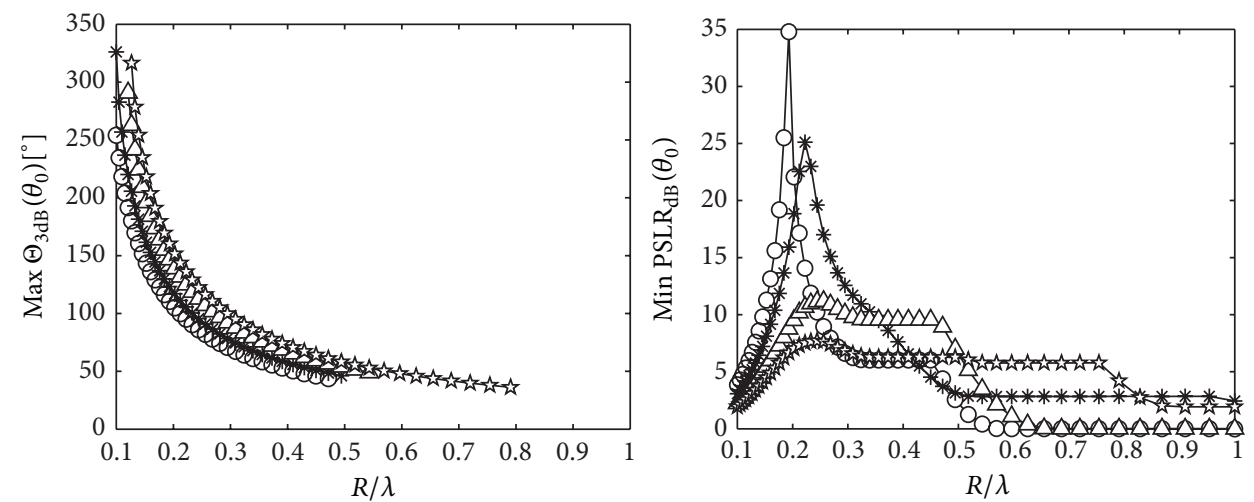

(d)

FIgURE 1: Continued. 

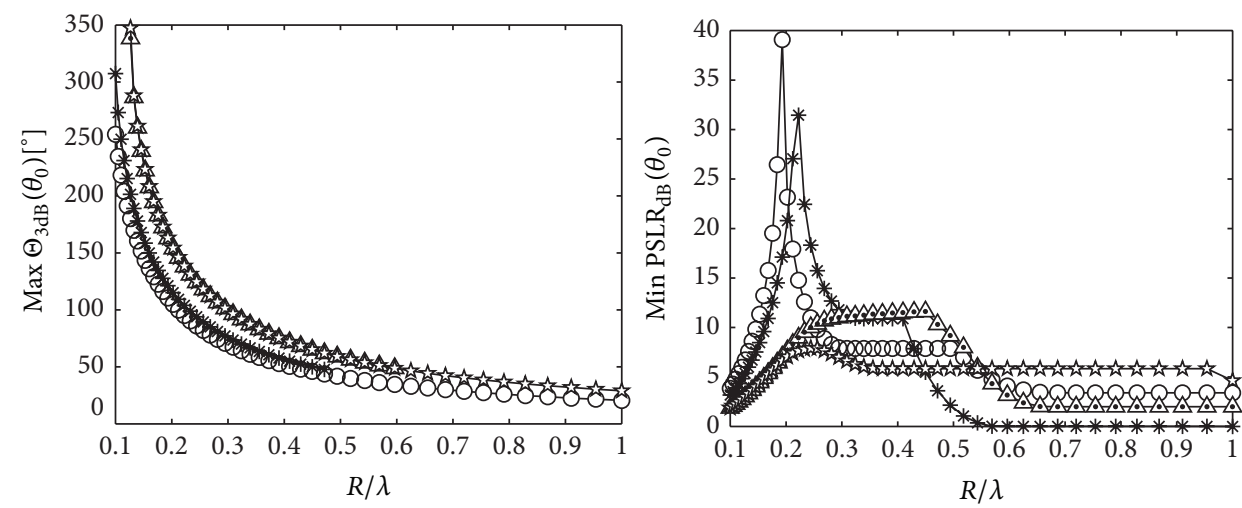

(e)
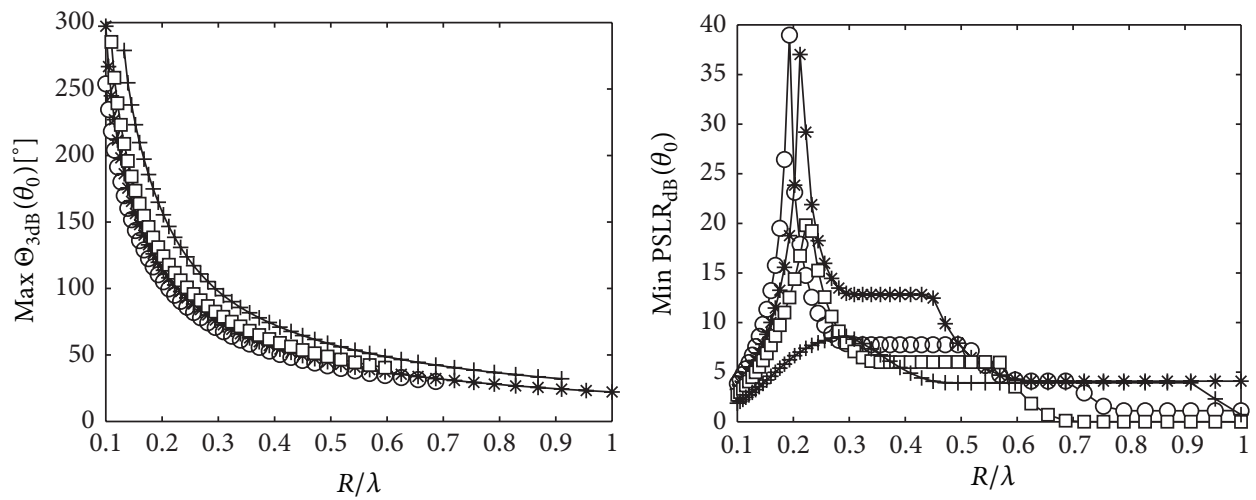

(f)
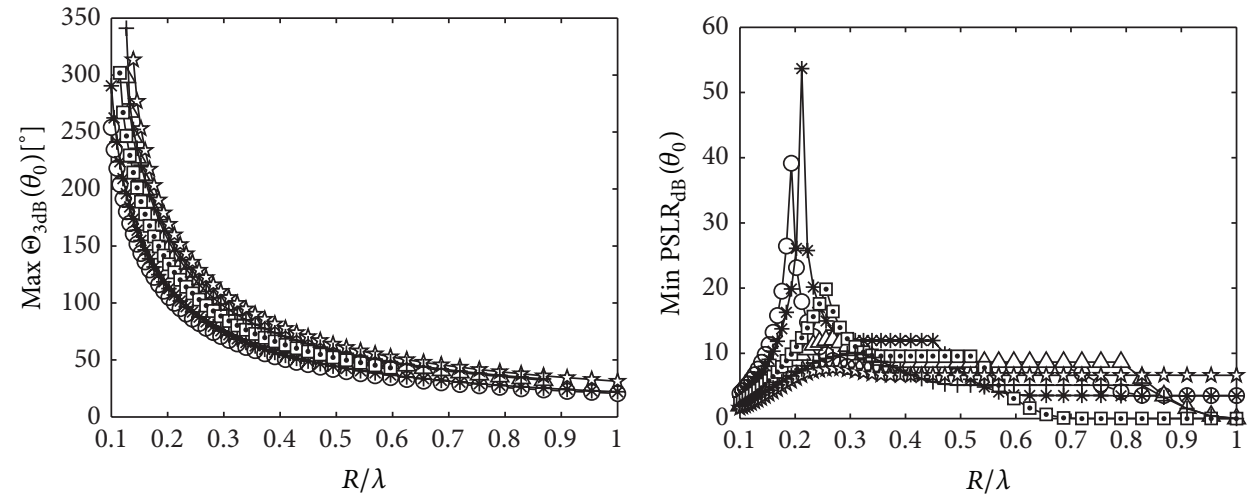

(g)

FIGURE 1: $3 \mathrm{~dB}$ aperture (left) and PSLR (right) for the configurations of Table 1: (a) 3-element configurations, (b) 4-element configurations, (c) 5-element configuration, (d) 6-element configurations, (e) 7-element configurations, (f) 8-element configurations, and (g) 9-element configurations. The legend is given in the lower row of Table 1.

is a delayed replica of the transmitted signal as well, but it is Doppler-shifted. Therefore, allowing an adaptive temporal filter to cancel only the zero Doppler echoes allows to remove the direct signal and its multipaths, without removing the target echo [4-6].

In the case where the direct signal is especially strong (e.g., when the passive radar receiver is very close to the transmitter of opportunity), the use of the temporal adaptivity following the antenna sidelobe attenuation of the direct signal might still be unsatisfactory. In this case spatially adaptive techniques may be considered to reject the disturbance, leading to an increased detection performance.

Antenna-based adaptive cancellation techniques provide rejection of the disturbance by means of adaptive beamforming. An estimate of the disturbance covariance matrix $\mathbf{M}$ is required to properly weight the received signals. Three approaches to adaptive cancellation are considered and discussed in the following three subsections, while in the last subsection the synthesis of the reference beam for the adaptive temporal filter is dealt with. 


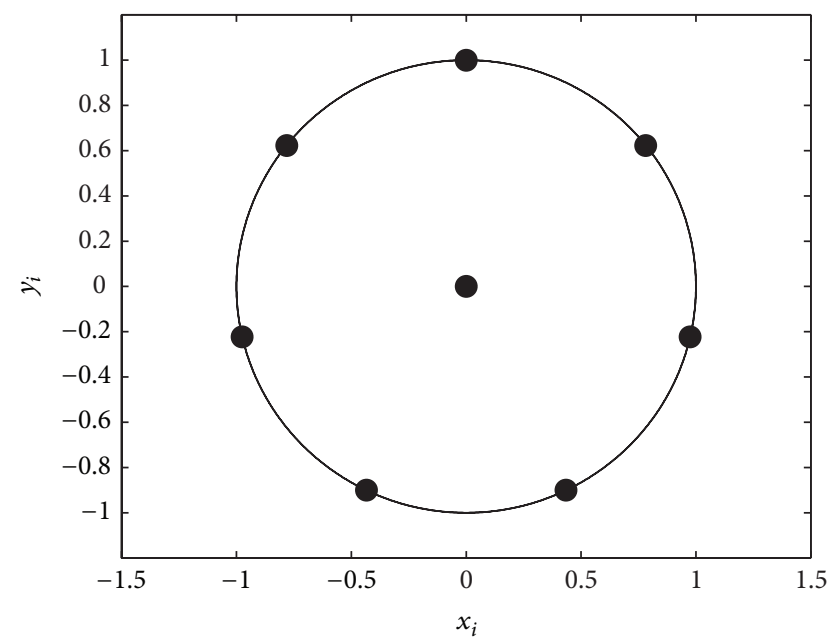

FIGURE 2: Circular configuration with central element for $N=8$.

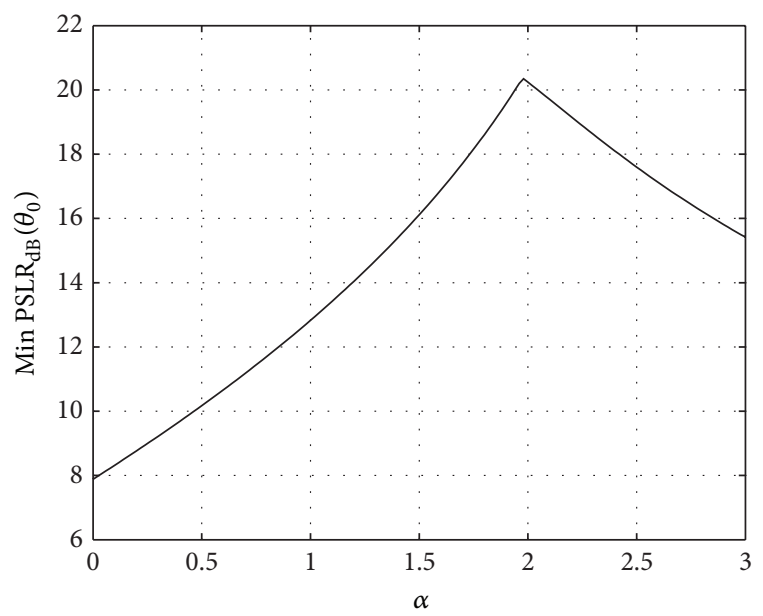

Figure 3: Trend of the PSLR as a function of the taper $\alpha$ for the configuration of Figure 2.

4.1. Optimum Filter. In case the disturbance covariance matrix $\mathbf{M}$ is known, the optimum weights vector, that leads to the maximum detection probability for a given false alarm rate, is given by [17]

$$
\mathbf{w}^{H}\left(\theta_{0}\right)=\mathbf{s}_{\boldsymbol{\theta}}^{H}\left(\theta_{0}\right) \mathbf{M}^{-1},
$$

where $\theta_{0}$ is the direction towards which the beam is pointed.

As an example, in Figure 5 (solid line), the array pattern obtained by weighting the signals received by the antennas of the selected configuration according to (16), for $\theta_{0}=120^{\circ}$, is represented, assuming that the disturbance covariance matrix M, but for a multiplicative constant, is given by

$$
\mathbf{M}=\mathbf{I}+\operatorname{INR}_{1} \mathbf{s}_{\boldsymbol{\theta}}\left(\theta_{I 1}\right) \mathbf{s}_{\boldsymbol{\theta}}^{H}\left(\theta_{I 1}\right)+\mathrm{INR}_{2} \mathbf{s}_{\boldsymbol{\theta}}\left(\theta_{I 2}\right) \mathbf{s}_{\boldsymbol{\theta}}^{H}\left(\theta_{I 2}\right),
$$

where $\mathbf{I}$ is the identity matrix, $\theta_{I 1}$ and $\theta_{I 2}$ are the DoAs of two independent narrow-band interfering signals, and $\mathrm{INR}_{1}$ and $\mathrm{INR}_{2}$ represent the signal-to-noise ratios of the interfering

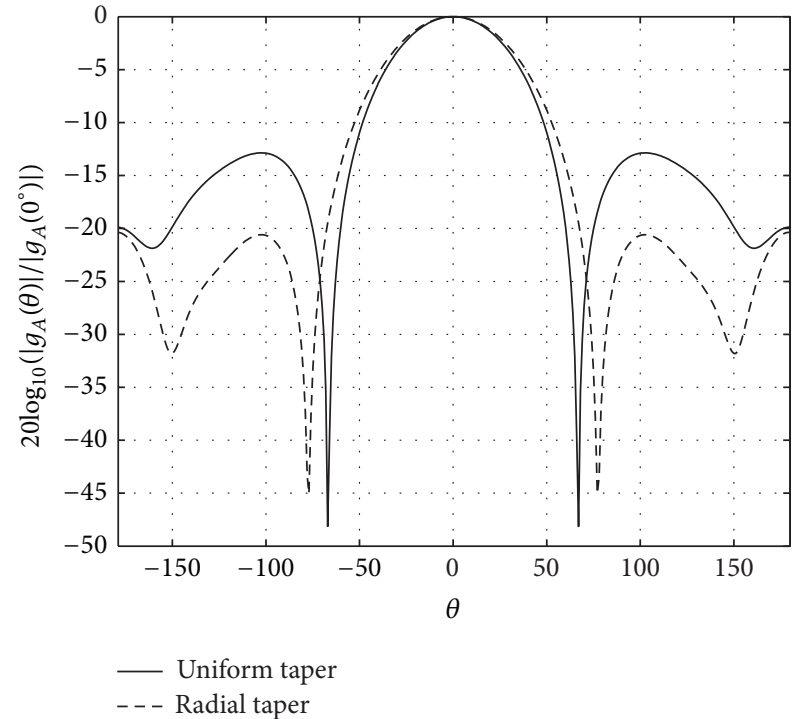

FIgURE 4: Array pattern with and without central element tapering for the configuration of Figure 2.

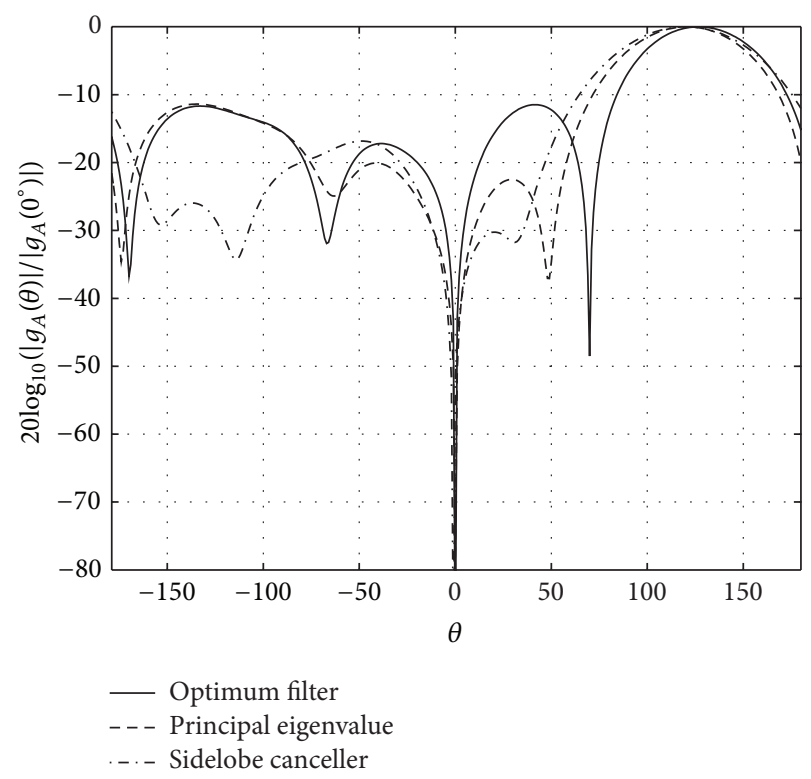

FIGURE 5: Examples of adaptive array patterns obtained by exploiting the optimum filter, the principal eigenvalue approach, and the Sidelobe Canceller for the case of two independent narrow-band interfering signals.

signals $\left(\theta_{I 1}=0^{\circ}, \theta_{I 2}=70^{\circ}, \mathrm{INR}_{1}=30 \mathrm{~dB}\right.$, and $\left.\mathrm{INR}_{2}=10 \mathrm{~dB}\right)$. The three components of the modeled disturbance covariance matrix represent the thermal noise, the direct signal, and a multipath replica of it. As it is apparent, the array pattern strongly attenuates the signals coming from the DoAs of both the direct signal and its multipath replica.

In practical applications, the covariance matrix $\mathbf{M}$ is replaced with its estimated version obtained from the received data, which include both the disturbance and the useful signal. This is quite appropriate in the PCL case given 


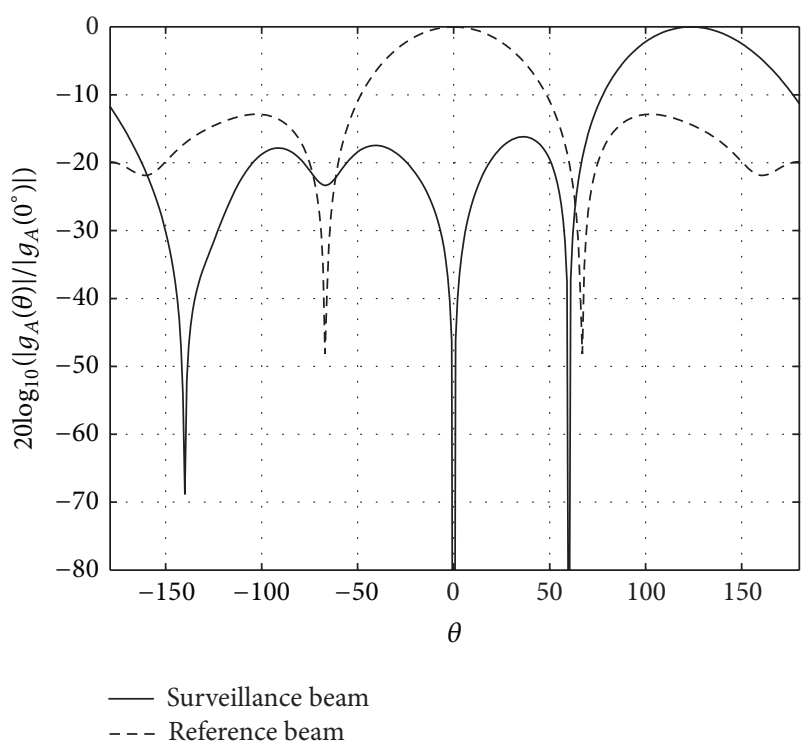

FIGURE 6: Example of surveillance beam and reference beam orthogonal to the surveillance one.

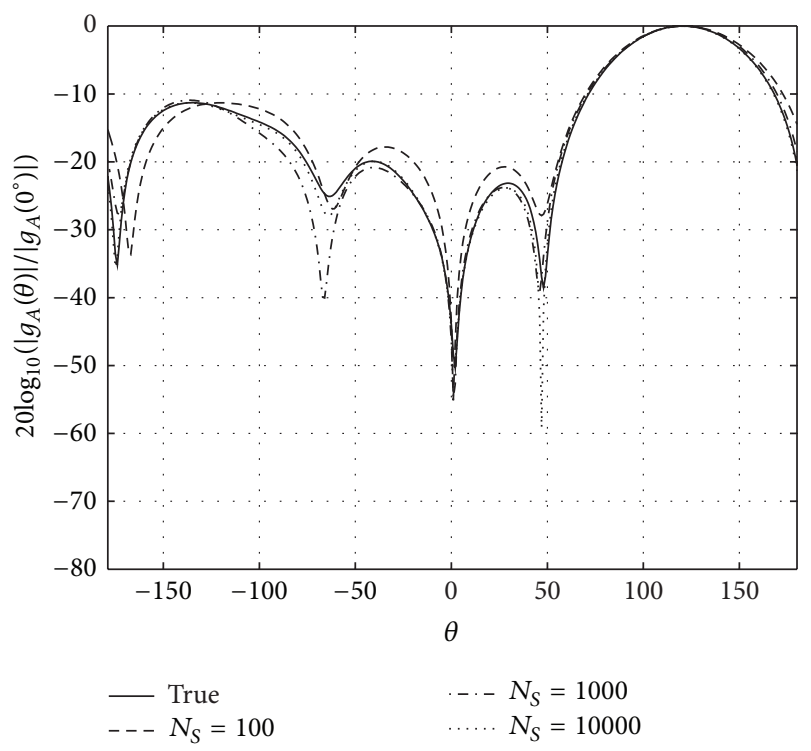

FIgURE 7: Pattern obtained by using the true and the estimated covariance matrix using $N_{S}=100, N_{S}=1000$, and $N_{S}=10000$ for the case of a single narrow-band interfering signal.

that the level of both the direct signal and its multipath reflections is well above that of the useful signal. After combining the received signals according to (16), an adaptive temporal cancellation might be performed only to cancel disturbance residuals not adequately suppressed by the spatial adaptive filter. Then, the standard 2D (range-Doppler) crosscorrelation function (2D-CCF) is evaluated, and a proper constant false alarm rate (CFAR) threshold is applied to obtain a detection map.

Notice that, when applying the optimum filter in the presence of a strong multipath reflection from a given direction,

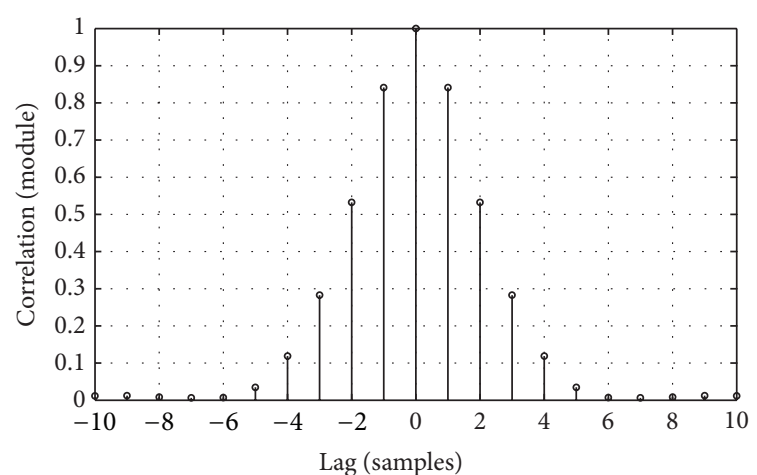

FIGURE 8: Example of autocorrelation function of an FM signal.

a null is imposed in the synthesized adaptive pattern at this direction. This also cancels all target echoes coming from the same DoA.

4.2. Principal Eigenvalue Approach. A way to circumvent the weakness of the optimum filter for targets at the same DoA of strong multipaths is to reduce the number of degrees of freedom (d.o.f.) available to the spatial adaptivity, so that only a single null can be imposed by the adaptive spatial filter. Since the direct signal is by far the strongest component of the overall disturbance, it is obvious that its effect dominates the spatial covariance matrix and specifically determines its principal eigenvector $\mathbf{q}_{\max }$. Therefore, by estimating only the principal eigenvector of the spatial disturbance covariance matrix, it is possible to strongly attenuate the direct signal, by projecting the target steering vector $\mathbf{s}_{\theta}(\theta)$ on the subspace orthogonal to such eigenvector. The resulting weights vector is given by

$$
\mathbf{w}_{\mathrm{PE}}^{H}\left(\theta_{0}\right)=\mathbf{s}_{\boldsymbol{\theta}}^{H}\left(\theta_{0}\right)\left(\mathbf{I}-\mathbf{q}_{\max } \mathbf{q}_{\max }^{H}\right) .
$$

Figure 5 (dashed line) shows the array pattern obtained by weighting the signals received by the antennas of the selected configuration according to (18), for $\theta_{0}=120^{\circ}$, assuming the same disturbance covariance matrix of the previous example. In this case, the array pattern strongly attenuates only the signals coming from the DoA of the direct signal, so that the clutter contributions have to be cancelled by the adaptive temporal filter. Finally, the 2D-CCF is evaluated, and the CFAR detection map is built.

4.3. Side Lobe Canceller (SLC). In case the disturbance is characterized by a single narrow-band interference, the array pattern obtained by using the optimum filter can be interpreted as the difference of a quiescent beam and a retrodirective beam, pointed in the direction of the disturbance. In particular, both beams are obtained by exploiting all the elements of the array.

A system based on the retrodirective beam, but at the same time characterized by a smaller number of d.o.f., can be obtained by resorting to the sidelobe canceller (SLC) concept [17], which, for the specific array configuration considered, can be exploited by using seven out of the eight elements of 


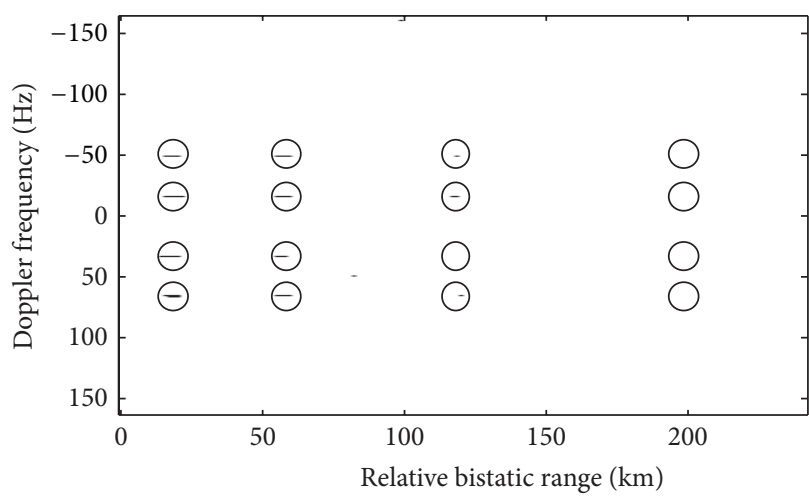

(a)

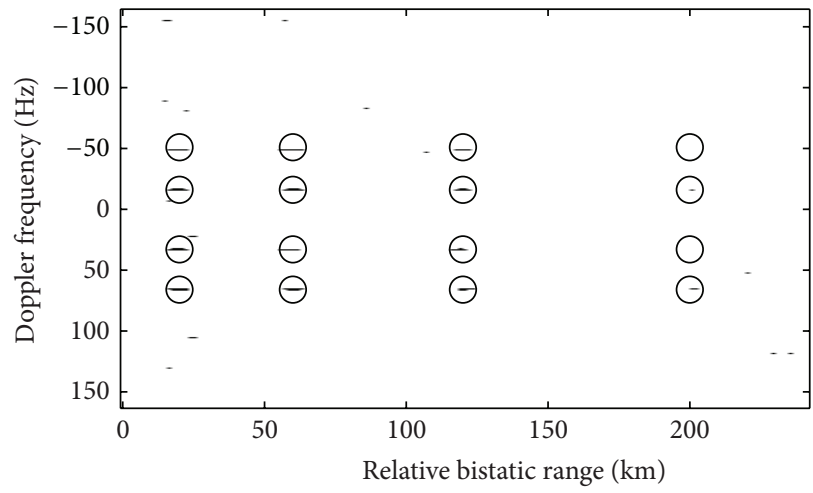

(c)

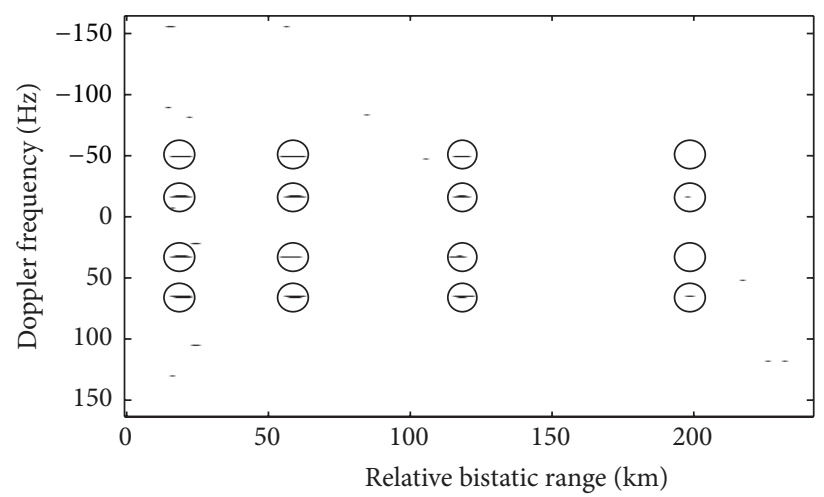

(b)

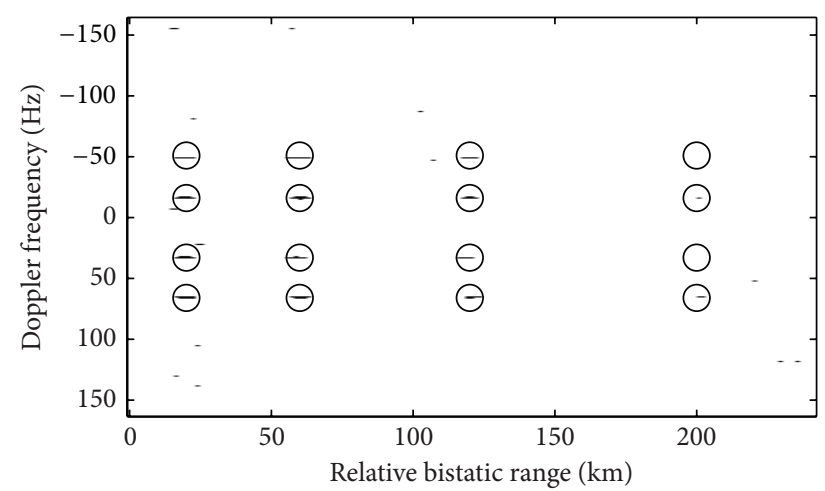

(d)

FIGURE 9: Detection results in the absence of stationary obstacles for (a) side lobe control by central element tapering (nonadaptive), (b) optimum filter, (c) principal eigenvalue approach, and (d) SLC.

the array to form the quiescent beam and the central element as an omnidirectional retrodirective beam.

Having defined the transformation matrix $\mathbf{T}$ as

$$
\mathbf{T}=\left[\begin{array}{ccccc}
s_{\boldsymbol{\theta} 1}^{*}\left(\theta_{0}\right) & s_{\boldsymbol{\theta} 2}^{*}\left(\theta_{0}\right) & \cdots & s_{\boldsymbol{\theta} 7}^{*}\left(\theta_{0}\right) & 0 \\
0 & 0 & \cdots & 0 & s_{\boldsymbol{\theta} 8}^{*}\left(\theta_{0}\right)
\end{array}\right]
$$

where $s_{\theta i}\left(\theta_{0}\right)$ is the $i$ th element of the target steering vector, corresponding $s_{\theta 8}\left(\theta_{0}\right)$ to the central element, the resulting weights vector for the SLC is given by

$$
\mathbf{w}_{\text {SLC }}^{H}\left(\theta_{0}\right)=\mathbf{s}_{\boldsymbol{\theta}}^{H}\left(\theta_{0}\right) \mathbf{T}^{H}\left(\mathbf{T M} \mathbf{T}^{H}\right)^{-1} \mathbf{T} .
$$

Notice that in this case a $2 \times 2$ matrix in place of an $8 \times 8$ matrix has to be inverted.

Figure 5 (dashed-dotted line) shows the array pattern obtained by weighting the signals received by the antennas of the selected configuration according to (20), for $\theta_{0}=$ $120^{\circ}$, assuming the same disturbance covariance matrix of the previous examples. The resulting antenna pattern is similar to the one obtained by exploiting the principal eigenvalue approach while the main lobe is only slightly broader.

Even in this case, the array pattern strongly attenuates only the signals coming from the DoA of the direct signal; therefore, the multipath reflections have to be cancelled by the adaptive temporal filter; then the 2D-CCF has to be evaluated, and a proper CFAR threshold has to be applied to detect potential targets over the range-Doppler plane.

4.4. Synthesis of the Reference Beam. In order to perform the adaptive temporal clutter cancellation, the direct signal is needed [4-6]. This can be obtained by synthesizing, in addition to the surveillance beam $\mathbf{w}_{\text {SURV }}$, a reference beam $\mathbf{w}_{\mathrm{REF}}$ pointed to the transmitter. In contrast to some passive radar systems based on directive antennas, where different physical antennas are used to collect the reference and the surveillance signals, the considered system uses the same antennas to collect both, by combining the received signals in different ways.

Rather than simply pointing the array beam to the desired direction, we synthesize a reference beam, orthogonal to the surveillance, such that

$$
\mathbf{w}_{\mathrm{REF}}^{H} \mathbf{w}_{\mathrm{SURV}}\left(\theta_{0}\right)=0 .
$$

Such an orthogonal reference beam may be obtained by using seven out of the eight elements of the array to point in the desired direction, while using the central element to satisfy 


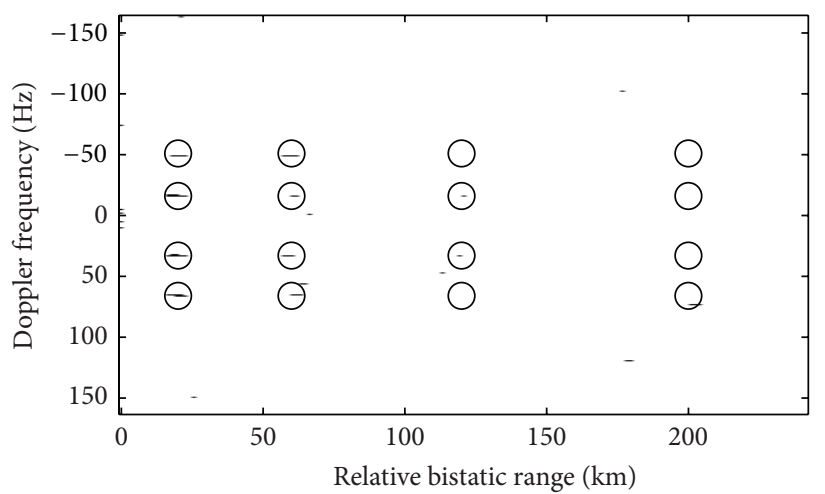

(a)

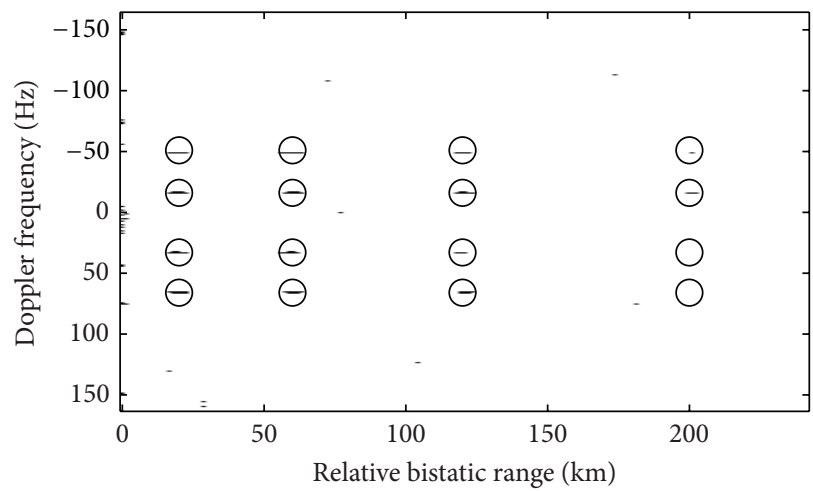

(c)

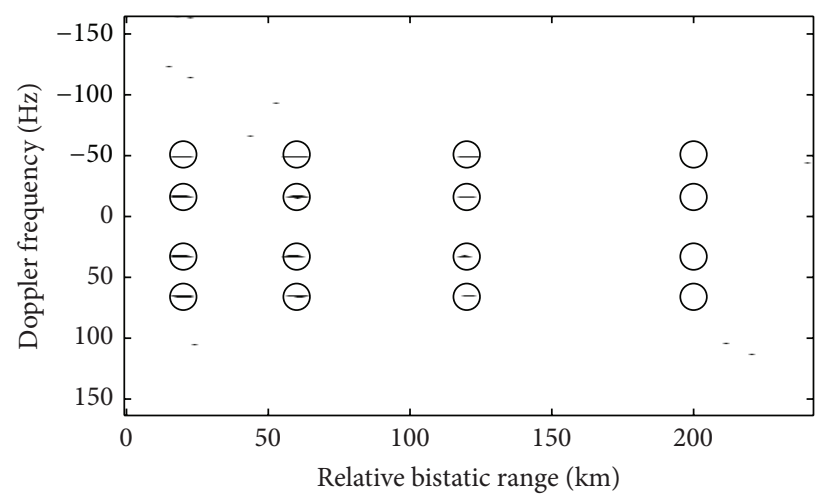

(b)

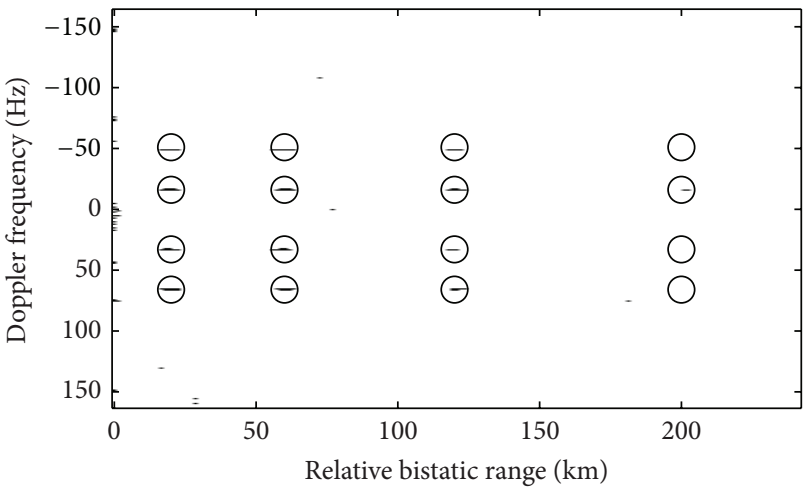

(d)

Figure 10: Detection results in the presence of a single stationary obstacle (DoA away from targets' DoAs) for (a) side lobe control by central element tapering (nonadaptive), (b) optimum filter (c) principal eigenvalue approach, and (d) SLC.

the condition given in (20). Denoting the surveillance and the reference beams as

$$
\begin{gathered}
\mathbf{w}_{\text {SURV }}\left(\theta_{0}\right)=\left[\begin{array}{c}
w_{\text {SURV } 1}\left(\theta_{0}\right) \\
w_{\text {SURV } 2}\left(\theta_{0}\right) \\
\vdots \\
w_{\text {SURV } 8}\left(\theta_{0}\right)
\end{array}\right]=\left[\begin{array}{c}
\mathbf{w}_{\text {SURV }}^{R}\left(\theta_{0}\right) \\
w_{\text {SURV } 8}\left(\theta_{0}\right)
\end{array}\right], \\
\mathbf{w}_{\text {REF }}=\left[\begin{array}{c}
s_{\theta 1}\left(\theta_{0}\right) \\
s_{\boldsymbol{\theta} 2}\left(\theta_{0}\right) \\
\vdots \\
s_{\boldsymbol{\theta} 7}\left(\theta_{0}\right) \\
\beta
\end{array}\right]=\left[\begin{array}{c}
\mathbf{s}_{\boldsymbol{\theta}}^{R}\left(\theta_{0}\right) \\
\beta
\end{array}\right],
\end{gathered}
$$

respectively, the coefficient $\beta$, which leads to an orthogonal reference beam, can be readily expressed as

$$
\beta=-\frac{\mathbf{w}_{\text {SURV }}^{R}{ }^{H}\left(\theta_{0}\right) \mathbf{s}_{\boldsymbol{\theta}}^{R}\left(\theta_{0}\right)}{w_{\text {SURV }}{ }^{*}\left(\theta_{0}\right)} .
$$

An example of reference beam orthogonal to the surveillance one is given in Figure 6.

\section{Simulation Results}

5.1. Methodology. In this section, a performance comparison is presented between the different approaches, with reference to the antenna array configuration above selected and sketched in Figure 2. To this purpose, a signal software simulator has been developed which is able to generate the signal received by a multichannel passive bistatic radar (PBR) under different conditions and exploiting different waveforms of opportunity. The developed simulator allows to specify the main parameters of both the transmitter and the receivers (the 8 receiving channels are supposed to be identical). Moreover, different bistatic geometries and clutter scenarios can be given as input data. Aiming at assessing the detection performance of the considered techniques, PBR scenarios with a relatively large number of targets have been considered.

In the following, we refer to an FM radio-based PBR, and the corresponding waveforms of opportunity are emulated according to the International Telecommunication Union (ITU) regulations [18].

The signals received by the eight elements of the array are then generated as the sum of several contributions, namely,

(i) the direct signal, which is an attenuated and delayed replica of the FM radio signal; 


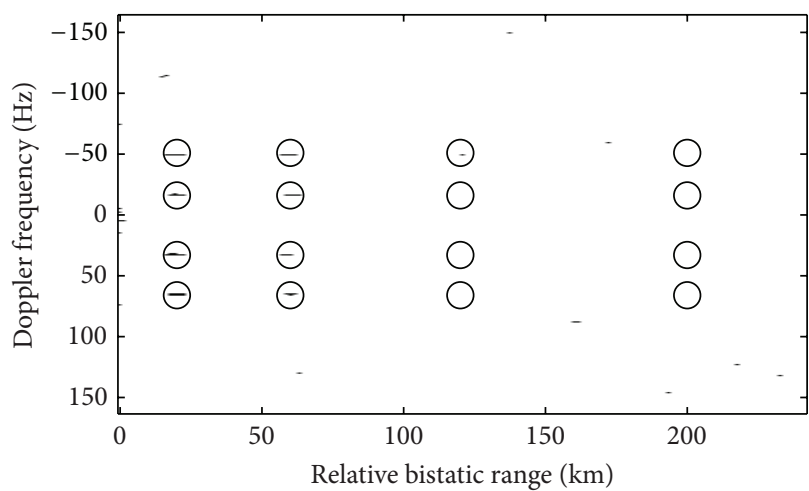

(a)

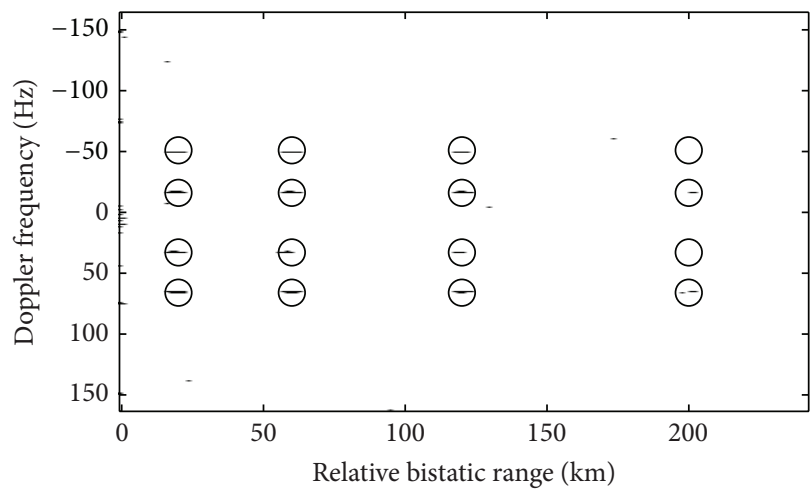

(c)

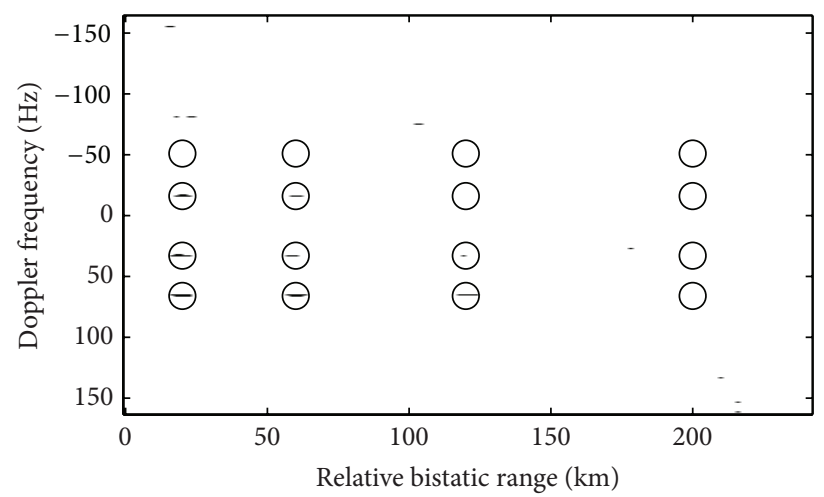

(b)

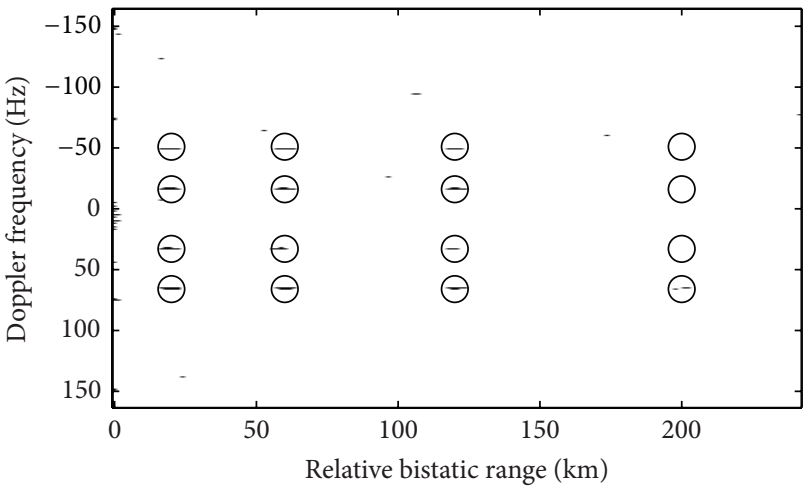

(d)

Figure 11: Detection results in the presence of a single stationary obstacle (DoA close to targets' DoAs) for (a) side lobe control by central element tapering (nonadaptive), (b) optimum filter, (c) principal eigenvalue approach, and (d) SLC.

(ii) the contributions of the stationary obstacles (clutter/multipath), which are still attenuated and delayed replicas of the FM radio signal;

(iii) the echoes from targets, which are attenuated, delayed, and Doppler-shifted replicas of the same FM radio signal;

(iv) the thermal noise, which is modeled as Additive White Gaussian Noise (AWGN).

The cascade of a spatial cancellation approach followed by a temporal adaptive cancellation filter is applied against the simulated data set. Specifically, the batch version of the ECA is adopted in the temporal dimension [5], while the spatial approach is selected among those discussed in the previous sections:

(i) side lobe control by central element tapering (nonadaptive);

(ii) optimum filter;

(iii) principal eigenvalue approach;

(iv) SLC.

Finally, the range-Doppler map is computed, and the detection performance is evaluated after the application of a conventional CFAR detection scheme. Several simulations have been run to provide averaged results.
5.2. Estimation of the Disturbance Covariance Matrix. As already mentioned, in case adaptive approaches are exploited, the disturbance covariance matrix has to be estimated from data. As the level of the useful signal is negligible compared to the level of the disturbance, the covariance matrix of the overall signal represents a good approximation of the disturbance covariance matrix.

Denoting $\mathbf{r}_{i}$ as the row vector containing $N_{S}$ samples of the signal received by the $i$ th antenna, let us define the $8 \times N_{S}$ matrix $\mathbf{R}$ as

$$
\mathbf{R}=\left[\begin{array}{c}
\mathbf{r}_{1} \\
\mathbf{r}_{2} \\
\vdots \\
\mathbf{r}_{i} \\
\vdots \\
\mathbf{r}_{8}
\end{array}\right] .
$$

An estimate of the covariance matrix is given by

$$
\widehat{\mathbf{M}}=\frac{1}{N_{S}} \mathbf{R R}^{H} .
$$

As far as the estimation accuracy is concerned, the larger the $N_{S}$ is, the more accurate the estimation of $\mathbf{M}$ is. In this specific application, $\mathbf{M}$ has to be estimated accurately enough that 


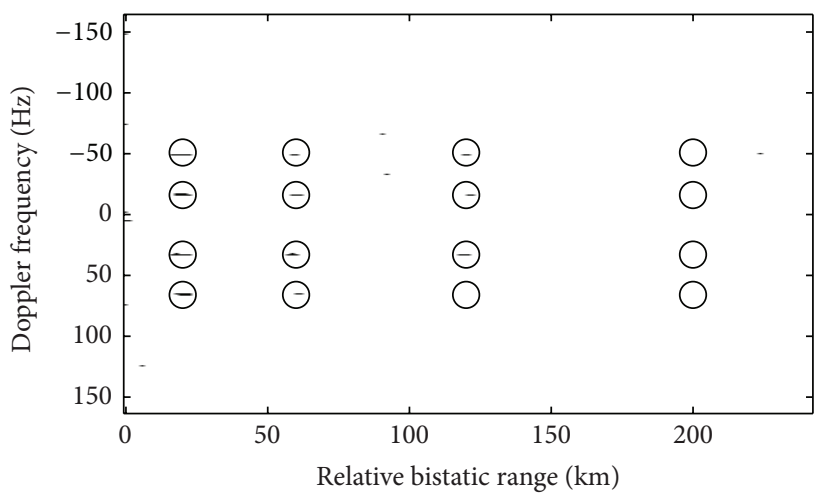

(a)

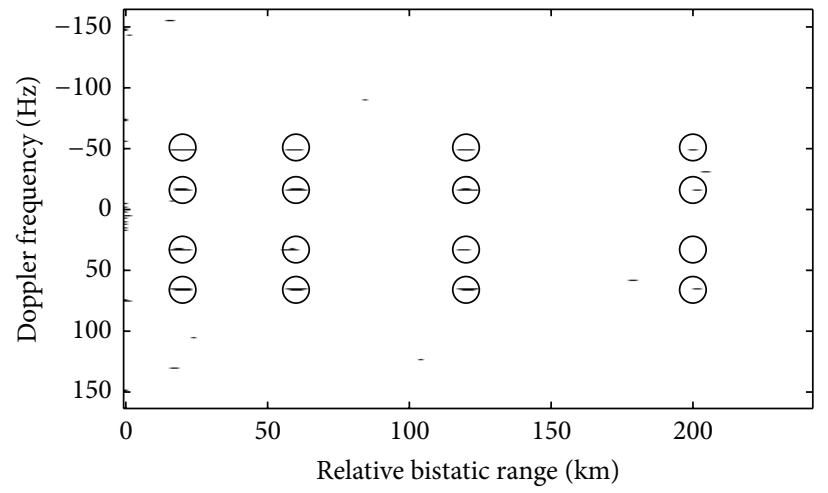

(c)

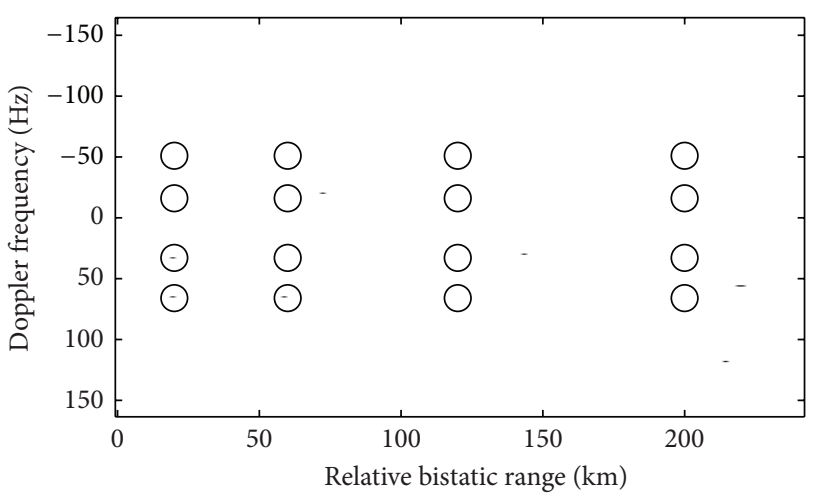

(b)

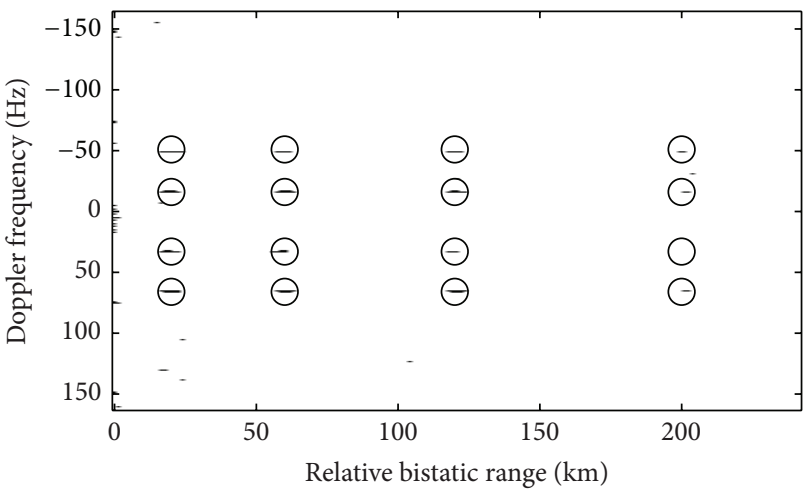

(d)

FIGURE 12: Detection results in the presence of several stationary obstacles for (a) side lobe control by central element tapering (nonadaptive), (b) optimum filter, (c) principal eigenvalue approach, and (d) SLC.

the resulting pattern does not differ significantly from the pattern obtained by using the true covariance matrix $\mathbf{M}$. In Figure 7, the pattern obtained for different values of $N_{S}$ and for the true covariance matrix is shown, in case the disturbance only includes the direct signal and the thermal noise, with power ratio equal to $75 \mathrm{~dB}$. In order to obtain a pattern very similar to the one obtained by using the true covariance matrix, at least $N_{S}=1000$ samples have to be used in the estimation. Notice that, for the considered FM radio-based $\mathrm{PBR}$, assuming a sampling frequency equal to $200 \mathrm{kHz}$, this number of samples would correspond to a signal fragment of $5 \mathrm{~ms}$ whose availability is easily guaranteed. Moreover, such duration assures the considered disturbance contribution to be quite stable during the estimation time due to the stationary characteristics in the spatial dimension.

Nevertheless, as consecutive samples are usually correlated (see an example of autocorrelation function of a received FM radio signal in Figure 8 ), $N_{S}$ can be reduced, if nonconsecutive samples are used.

5.3. Detection Performance. In the case study reported in this paragraph, sixteen targets have been injected in the simulated data with target-receiver distance ranging from 10 to $100 \mathrm{~km}$, DoAs comprised between $150^{\circ}$ and $180^{\circ}$, radial velocities in the range $[-150,200] \mathrm{m} / \mathrm{s}$, and signal-to-noise ratios between -35 and $-55 \mathrm{~dB}$. Different clutter scenarios are then considered.

Figures 9(a)-9(d) report the detection results obtained for different processing schemes in the absence of stationary obstacles. As is apparent, the three spatial adaptive approaches (Figures 9(b)-9(d)) yield comparable performance allowing the detection of fourteen out of sixteen targets (circles indicate the injected targets positions over the range-Doppler plane). Only eleven out of sixteen targets are, instead, detected by using the nonadaptive approach (see Figure 9(a)). In the latter case, in fact, the poor detection performance is due to the fact that the direct signal, received with power level $70 \mathrm{~dB}$ above thermal noise, is not attenuated enough by the array pattern of the surveillance beam; therefore, the temporal adaptive cancellation of the residual direct signal implies the cancellation of part of the useful signal as well.

Similar performances are observed in case a single stationary obstacle with power level $30 \mathrm{~dB}$ above thermal noise, whose DoA is away from the targets' DoAs, is introduced in the scene (see Figure 10).

The detection performances of the three adaptive schemes are no longer similar when the DoA of the stationary scatterer is close to the DoAs of some of the targets (Figure 11). In that case, as is expected, optimum filter experiences some detection losses, specifically failing 
in detecting targets with DoAs close to the DOA of the multipath return. In particular, Figure 11(b) shows that the optimum filter does not detect a target characterized by a quite short bistatic range/high bistatic RCS because its DoA is close to that of the obstacle.

Figure 12 reports the detection results obtained for a simulated scenario containing three stationary obstacles with clutter-to-noise ratios ranging between 25 and $30 \mathrm{~dB}$. As it is apparent and as it has been mentioned, the optimum filter yields an effective removal of all the undesired disturbance contributions which, however, implies also many of the injected targets to be lost. In contrast, the exploitation of a reduced number of d.o.f., as required by the principal eigenvalue approach and the SLC, allows the effective cancellation of the sole main disturbance contribution typically represented by the direct signal. However, the clutter/multipath contributions can be effectively cancelled by the temporal adaptive filter. This prevents the undesired target cancellation at the DoA of the strong multipaths thus yielding remarkable detection performance.

As a final remark, we would like to point out that the cascading of an antenna-based adaptive technique and a temporal adaptive cancellation filter makes the system robust also to the presence of strong interferences in the exploited signal frequency band. Notice that the ECA is not effective against such disturbance contributions since, as long as it is concerned, this filter looks for delayed replicas of the exploited signal of opportunity. However, cochannel interferences are typically observed in practical passive radar applications based on broadcast transmissions due to the frequency reuse over neighbouring areas.

\section{Conclusions}

A suitable array configuration for passive radar application has been identified and optimized by designing a specific tapering solution. The selected antenna array allows both to steer the surveillance beam in order to cover a wide surveillance area and to strongly attenuate the undesired direct signal in the surveillance channel, thus preserving the useful dynamic range of the receiver. Three spatial adaptive approaches have also been investigated and compared, showing their effectiveness in removing the undesired contributions from the surveillance PBR signals. A comparative performance analysis has been carried out among different processing schemes with reference to a simulated casestudy. It has been shown that the most effective solution is obtained by resorting to the principal eigenvalue approach or to the SLC for direct signal spatial suppression, and then exploiting a temporal adaptive cancellation filter to remove both direct signal residuals and its multipath reflections in the surveillance channel. In particular, the SLC solution is likely to be preferred due to its limited complexity.

\section{Acknowledgment}

At the time the major part of this work was done, M. Villano was developing his master's thesis within the DIET
Department, University of Rome "La Sapienza", Italy, which fully funded this work.

\section{References}

[1] "Special issue on passive radar systems-IEE proceedings on radar," Sonar and Navigation, vol. 152, no. 3, pp. 106-223, 2005.

[2] P. E. Howland, D. Maksimiuk, and G. Reitsma, "FM radio based bistatic radar," IEE Proceedings on Radar, Sonar and Navigation, vol. 152, no. 3, pp. 107-115, 2005.

[3] H. D. Griffiths and C. J. Baker, "Passive coherent location radar systems. Part 1: performance prediction," IEE Proceedings on Radar, Sonar and Navigation, vol. 152, no. 3, pp. 153-159, 2005.

[4] R. Cardinali, F. Colone, C. Ferretti, and P. Lombardo, "Comparison of clutter and multipath cancellation techniques for passive radar," in Proceedings of the IEEE Radar Conference, pp. 469474, Boston, Mass, USA, April 2007.

[5] F. Colone, D. W. O’Hagan, P. Lombardo, and C. J. Baker, “A multistage processing algorithm for disturbance removal and target detection in passive bistatic radar," IEEE Transactions on Aerospace and Electronic Systems, vol. 45, no. 2, pp. 698-722, 2009.

[6] F. Colone, R. Cardinali, and P. Lombardo, "Cancellation of clutter and multipath in passive radar using a sequential approach," in Proceedings of the IEEE Radar Conference, pp. 393399, Verona, NY, USA, April 2006.

[7] M. Malanowski and K. Kulpa, "Digital beamforming for passive coherent location radar," in Proceedings of the IEEE Radar Conference (RADAR '08), Rome, Italy, May 2008.

[8] P. Knott and U. R. O. Nickel, "Design and development of a V-shaped printed dipole antenna array for passive radar," in Proceedings of the 5th European Conference on Antennas and Propagation (EUCAP '11), pp. 961-964, Rome, Italy, April 2011.

[9] F. Belfiori, S. Monni, W. van Rossum, and P. Hoogeboom, "Antenna array characterisation and signal processing for an FM radio-based passive coherent location radar system," IET Radar, Sonar \& Navigation, vol. 6, no. 8, pp. 687-696, 2012.

[10] M. Villano, F. Colone, and P. Lombardo, "Adaptive clutter suppression in passive phased array radar," in Proceedings of the International Radar Symposium (IRS '09), pp. 343-347, Hamburg, Germany, September 2009.

[11] R. Zemmari, U. Nickel, and W.-D. Wirth, "GSM passive radar for medium range surveillance," in Proceedings of the European Radar Conference (EuRAD '09), pp. 49-52, Rome, Italy, October 2009.

[12] J. Zhu, Y. Hong, and L. Tao, "Adaptive beamforming passive radar based on FM radio transmitter," in Proceedings of the IET International Conference on Radar Systems, Edinburgh, UK, October 2007.

[13] G. Fabrizio, F. Colone, P. Lombardo, and A. Farina, "Adaptive beamforming for high-frequency over-the-horizon passive radar," IET Radar, Sonar and Navigation, vol. 3, no. 4, pp. 384405, 2009.

[14] J.-H. Deng, J.-K. Hwang, C.-Y. Lin, and S.-M. Liao, "Adaptive space-time beamforming technique for passive radar system with ultra low signal to interference ratio," in Proceedings of the IEEE International Conference on Wireless Information Technology and Systems (ICWITS '10), September 2010.

[15] F. Colone, R. Cardinali, P. Lombardo et al., "Space-time constant modulus algorithm for multipath removal on the reference signal exploited by passive bistatic radar," IET Radar, Sonar and Navigation, vol. 3, no. 3, pp. 253-264, 2009. 
[16] A. Farina, P. Lombardo, and L. Ortenzi, "A unified approach to adaptive radar processing with general antenna array configuration," Signal Processing, vol. 84, no. 9, pp. 1593-1623, 2004.

[17] A. Farina, Antenna-Based Signal Processing Techniques for Radar Systems, Artech House, Norwood, NJ, USA.

[18] A. Lauri, F. Colone, R. Cardinali, C. Bongioanni, and P. Lombardo, "Analysis and emulation of FM radio signals for passive radar," in Proceedings of the IEEE Aerospace Conference, pp. 2170-2179, Big Sky, Mont, USA, March 2007. 

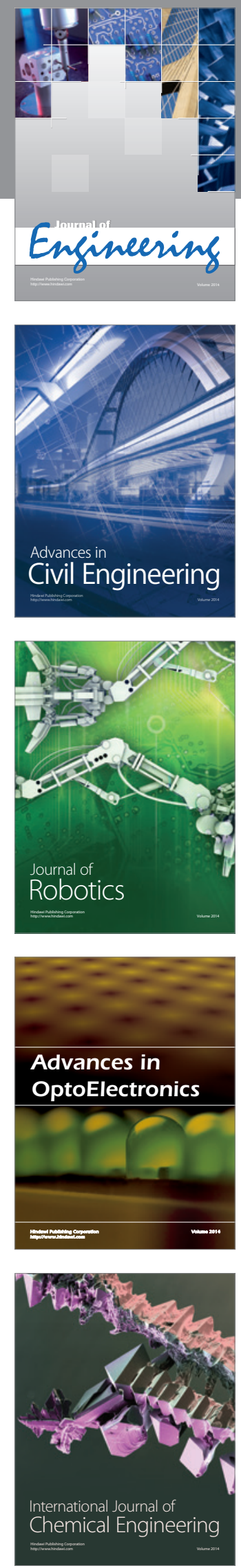

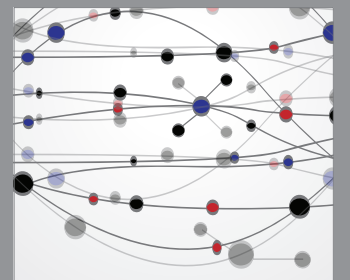

The Scientific World Journal
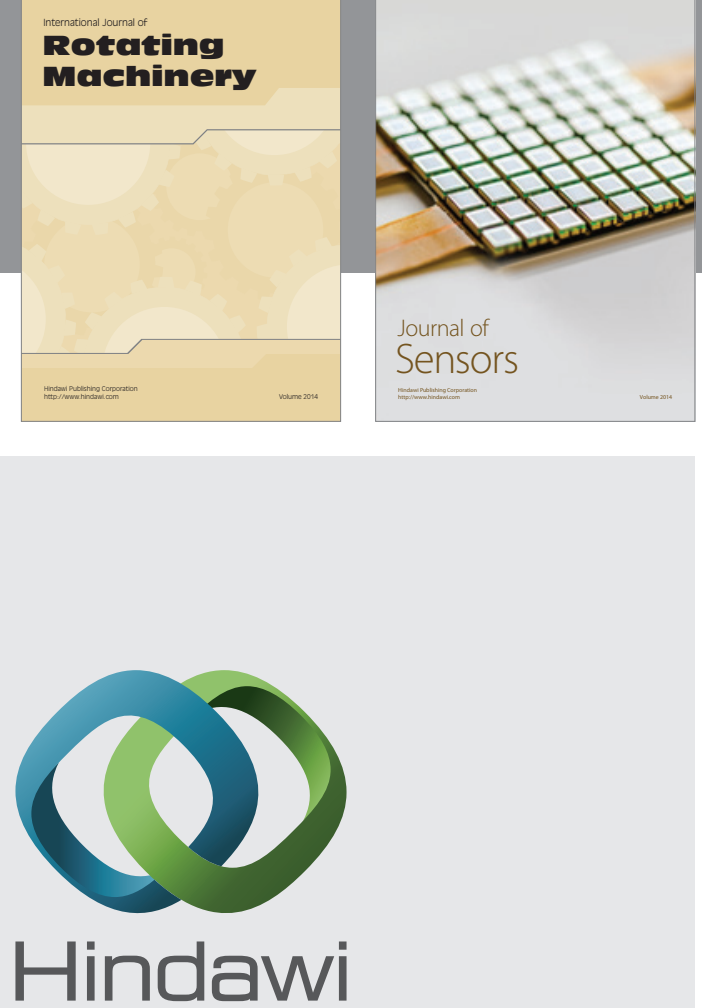

Submit your manuscripts at http://www.hindawi.com
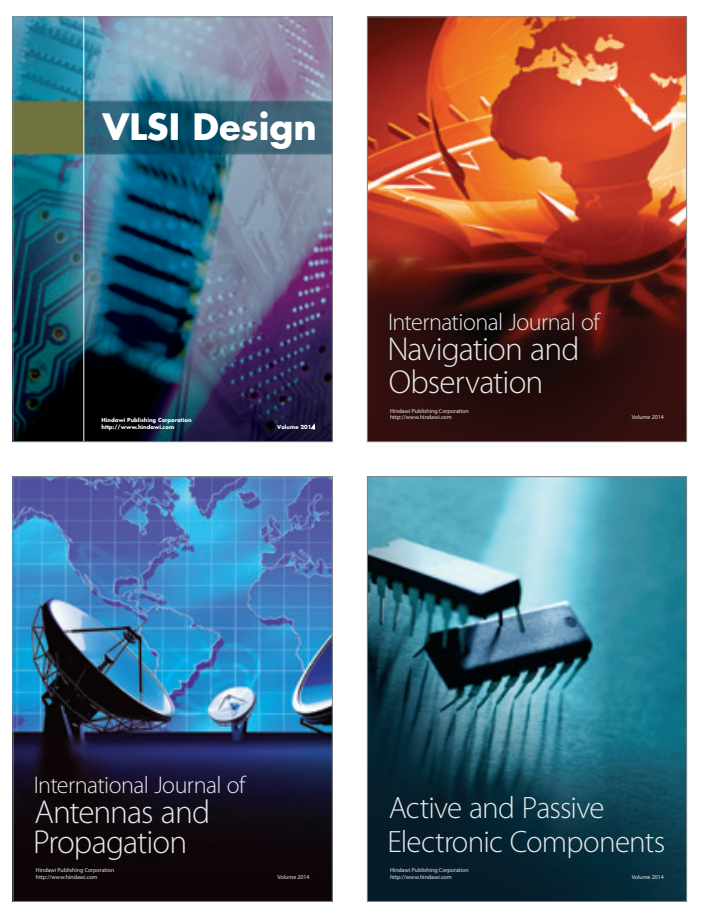
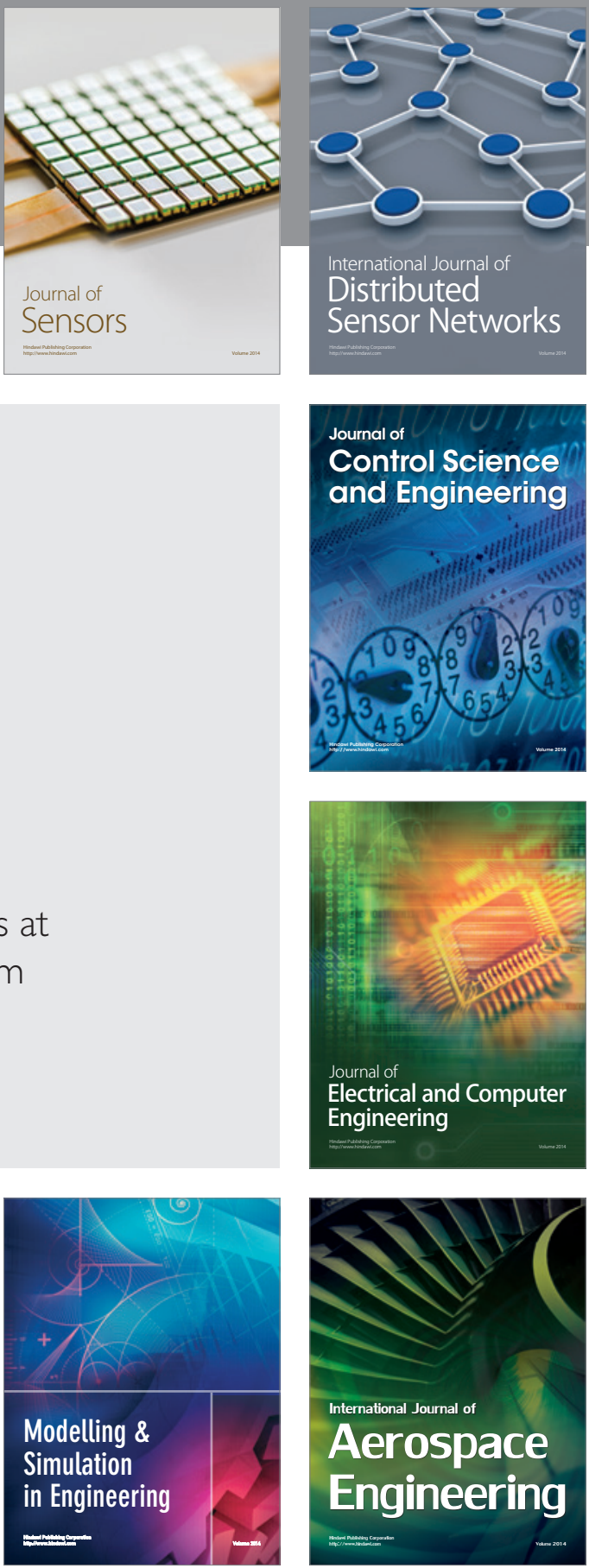

Journal of

Control Science

and Engineering
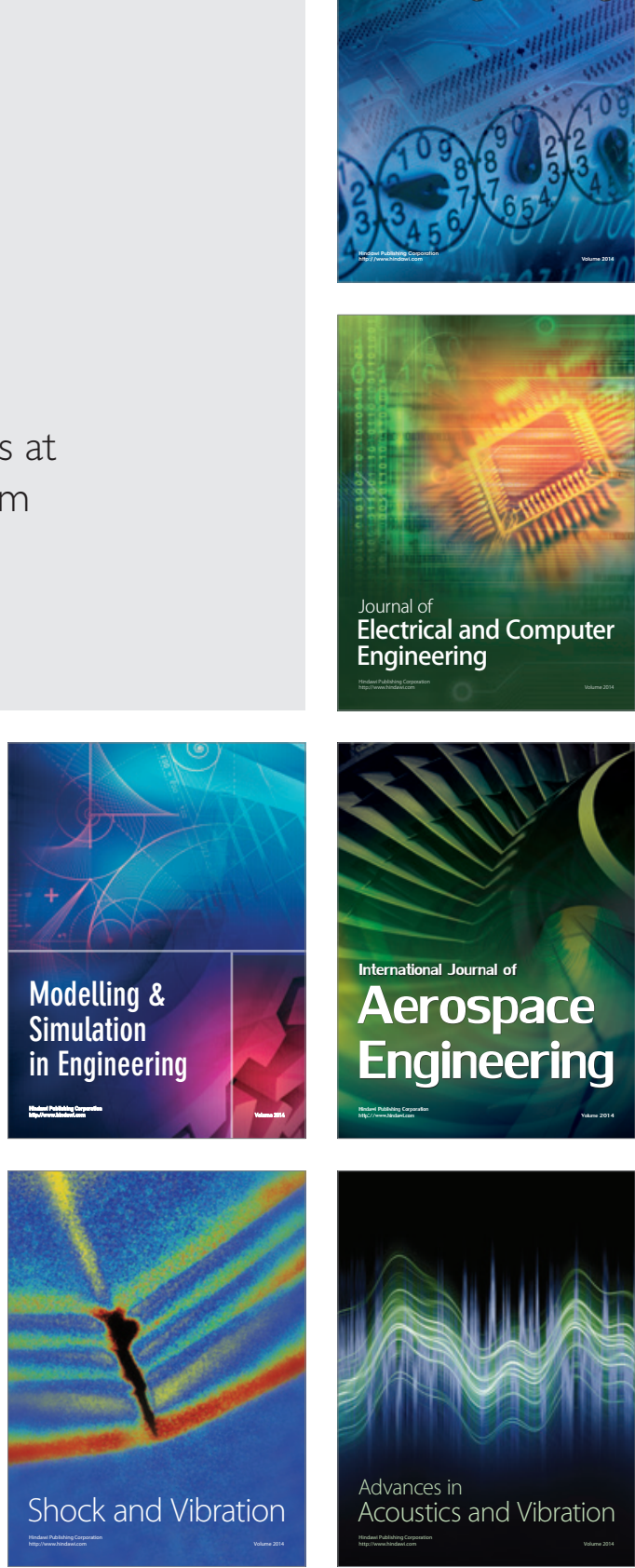